\title{
The Cretaceous Alkaline Dyke Swarm in the Central Segment of the Asunción Rift, Eastern Paraguay: Its Regional Distribution, Mechanism of Emplacement, and Tectonic Significance
}

\author{
Victor F. Velázquez, ${ }^{1}$ Claudio Riccomini, ${ }^{2}$ Celso de Barros Gomes, ${ }^{2}$ and Jason Kirk ${ }^{2}$ \\ ${ }^{1}$ Escola de Artes, Ciências e Humanidades, Universidade de São Paulo, Rua Arlindo Béttio 1000, Ermelino Matarazzo, \\ 03828-000 São Paulo, SP, Brazil \\ ${ }^{2}$ Instituto de Geociências, Universidade de São Paulo, Rua do Lago 562, 05508-080 São Paulo, SP, Brazil
}

Correspondence should be addressed to Victor F. Velázquez, vvf@usp.br

Received 21 July 2010; Revised 16 November 2010; Accepted 19 February 2011

Academic Editor: Jan Veizer

Copyright (C) 2011 Victor F. Velázquez et al. This is an open access article distributed under the Creative Commons Attribution License, which permits unrestricted use, distribution, and reproduction in any medium, provided the original work is properly cited.

\begin{abstract}
A structural analysis of Cretaceous alkaline dykes swarm associated with the central segment of the Asunción Rift is reported here. Dykes are generally single near-vertical tabular bodies, less than $5 \mathrm{~m}$ wide, although multiple and composite intrusions also occur. Many of these small bodies have been emplaced into Paleozoic sedimentary rocks and exhibit a regional NW-SE orientation pattern. Petrographical and geochemical data allow recognition of two different lineages of potassic dykes: a silicaundersaturated suite ranging from basanite to phonolite (B-P) and a silica-saturated suite ranging from alkali basalt to trachyte $(\mathrm{AB}-\mathrm{T})$. The morphological features, the regional en-échelon distribution, and the NW-SE orientation pattern suggest that the dykes were injected along fractures and faults, under a transtensional tectonic regime with $\sigma 1 \mathrm{NW} /$ horizontal, $\sigma 2 /$ vertical, and $\sigma 3$ $\mathrm{NE}$ /horizontal. Detailed analysis, combining dyke petrography, orientation pattern, and relative chronology reveals a rotation from WNW toward NNW during dyke emplacement. In terms of the paleostress field orientation, the evidence indicates that the dykes were diachronically formed under a similar stress condition. Finally, the pattern of orientation documented for the Cretaceous alkaline dykes of the Asunción Rift is consistent, temporally and spatially, with the phases of regional deformation that occurred during the process of the Atlantic Ocean opening.
\end{abstract}

\section{Introduction}

In many volcanic systems, dyke intrusions of variable size and composition are often considered the main channel feeders and represent one of the more important vertical transfer routes of mantle-derived molten material through the lithosphere to the upper crust. In general, dykes occur in diverse geological times and tectonic settings; however, the vast majority of the dyke swarms on continental areas are of the Proterozoic or Late Phanerozoic age [1]. Although their abundance in the crust is less expressive than continental flood basalts and large granitic masses, dykes are excellent tracers for many geological processes. Over the last two decades, numerous geochemical studies have been carried out on these small vertical tabular-like bodies with the purpose of better understanding the cause and nature of partial melting and magmatic differentiation processes. Consequently, many of the predictions made about the thermodynamic behavior and the conditions that govern the lower and upper mantle are based on the isotope and trace element signatures of dykes [2-4]. Dykes are largely used to examine the relationship involving elastic brittle-fluidhost rocks and magma transport. These studies are mainly targeted at determining the regional paleostress fields and the active mechanism $[4,5]$. A theoretical and applied approach about the emplacement mechanism and propagation of individual dykes is well documented in the synoptic paper of Anderson [6].

Eastern Paraguay is an intracratonic region situated in the westernmost border of the Paraná basin. During Early to Late Cretaceous time, this region was affected by an important tectonomagmatic event, the Paraná-Etendeka 
large igneous province (cf. [7]) related to the opening of the South Atlantic Ocean, which caused a series of alkaline magmatic episodes $[8,9]$. Although the distribution of these alkaline bodies is quite widespread around the Paraná basin (Figure 1), in the Eastern Paraguay region, they are confined to distinct well-exposed areas. In the northern (Rio Apa) and northeastern (Amambay) provinces, the alkaline rocks show similar ages of $138.9 \pm 0.7 \mathrm{Ma}$ (cf. [10]), predating the tholeiitic lavas of the Serra Geral Formation (133-130 Ma, cf. [11-15]). However, in the Central-Eastern (Central) and southern (Misiones) provinces, they are younger than the basaltic rocks, $126.4 \pm 0.4 \mathrm{Ma}$ and $118.3 \pm 1.6 \mathrm{Ma}$, respectively (cf. [10]).

One of the most conspicuous occurrences of alkaline rocks in Eastern Paraguay is represented by the Central Province. Geological and geophysical data indicate that the Asunción Rift development, in Cretaceous time, was responsible for multiple diachronous events of potassic alkaline magmatism (Central Province, cf. [10, 16, 17]). The mode of occurrence of the numerous bodies is quite variable. Intrusive formations are mainly represented by stocks of various dimensions; on the other hand, the extrusive units comprise essentially lava flows, domes, and plugs. Nevertheless, the most significant magmatic event of the province is represented by the hypabyssal rocks, occurring largely as individual dykes, which exhibit a wide variation in composition, texture, and size.

The good exposure of the outcrops offers an excellent opportunity to examine in detail the contact relationships, the morphological feature of the walls surface, and the orientation pattern of the dykes. Based on these data, this paper discusses the mechanism of emplacement, as well as the regional distribution of the paleostress field at the time of intrusion.

\section{Geological Setting}

The central area of Eastern Paraguay is characterized by an important tectonomagmatic-sedimentary event of Early Cretaceous age [18-20]. The major faulting zone is represented by the Asunción Rift [21], a tectonic feature of roughly $200 \mathrm{~km}$ length and $25-45 \mathrm{~km}$ wide, with NW-SE general orientation (Figure 2). According to Velázquez et al. [17] and Riccomini et al. [22], the rift consists of three different segments. The western segment, with a well-defined NWSE-trend, extends more than $90 \mathrm{~km}$ between the localities of Benjamin Aceval and Paraguarí, and it comprises an extensive Cenozoic sedimentary deposit and several Tertiary intrusive bodies of sodic ultra-alkaline rocks (Asunción Province, cf. [17, 20, 23]). The central E-W-trending segment measures approximately $70 \mathrm{~km}$, extends from the town of Paraguarí to the locality of Villarrica, and represents the region of major potassic alkaline magmatism. Largely, those rocks cut siliciclastic deposits of Silurian and Permian ages and locally the Early Cretaceous aeolian deposits of the Misiones Formation. Finally, the less defined NW-SEtrending eastern segment, with about $40 \mathrm{~km}$ of extension, is developed from the locality of Villarrica until the Ybytyruzú mountains. In the last area, the potassic alkaline rocks intrude both the Cretaceous aeolian deposits of the Misiones Formation and the overlying tholeiitic flows of the Paraná basin volcanism.

From a geodynamic viewpoint, at least two tectonic episodes of importance led to the current shape of the relief in the area. The first event, of Early Cretaceous age, which was induced by an NE-SW extensional tectonic regime, provoked major graben faulting and expressive potassic alkaline magmatism. The second one was also of extensional regime, with the major period of faulting taking place in the Paleocene. However, it continues until today causing small seismic movements of low amplitude in the region [24]. Possibly, a generalized lithospheric thinning and the emplacement of a hot mantle closer to the upper crust were responsible for the significant changes of the geothermic gradient occurred in this epoch. A detailed study of fission tracks in Silurian deposits and Cretaceous alkaline rocks (cf. [25]) indicates two different periods of thermal activity for the whole area, between 90-60 Ma and 60$10 \mathrm{Ma}$. These cooling ages are significantly younger than the tectonomagmatic event of the Central Province, but, in part, are consistent with the intrusion of the nephelinitic alkaline rocks of the Asunción Province, which mainly formed at $58.7 \pm 2.4 \mathrm{Ma}$ (cf. [10]). According to Riccomini et al. [20], the last period of time corresponds to an important tectonic phase, with generation of deep faulting that served as conduit for the mantle material to migrate to the surface of the crust.

\section{Morphology, Occurrence and Field Relations}

The potassic alkaline dyke intrusions are well exposed throughout a large section of the central segment of the Asunción Rift. More than 200 bodies of mafic, intermediate, and felsic dykes are randomly distributed in that region. The largest continuous exposures of individual dykes are found in the Sapucai district $[16,26]$ (Figure 3). Other important outcrops are confined to near the villages of Potero Ybaté and Gral. Bernardino Caballero is not so far from the town of Sapucai. Some dykes were also recorded in the Ybytymí region.

Most of the dykes show a continuous tabular aspect or are composed of several separate segments, resulting in an apparent sinuous intrusion. In both cases, they occur essentially as single intrusions, but multiple and composite injections are also present. Here, the multiple term is used to characterize a repeated injection of dyke with the same or similar compositions (alkaline basalt, trachybasalt, and trachyandesite), and composite when the repeated injections are of different compositions (alkaline basalt, tephrite, and phonolite).

The width of the dykes can vary from $0.15 \mathrm{~m}$ to $10 \mathrm{~m}$, but in most of the cases is between $0.30-3 \mathrm{~m}$; in general, the length cannot be traced for more than a few kilometers. Regionally, the dykes cross-cut Paleozoic deposits. In the Sapucai and Potrero Ybaté regions, they are intruded into lavas and plugs of alkaline rocks. The dyke-wall rock contact is usually vertical to subvertical, with chilled margins 
well preserved, and with prevalence for normal dilatation. Oblique relative displacement between the walls, in a dextral or sinistral sense, was also observed.

In a regional distribution, the dykes show a parallel to subparallel orientation and, in some cases, an en-échelon arrangement (Figure 3). However, there are localities where the orientation is less regular, indicating a significant change of direction and trajectory. In this case, the intrusion relationship is complex. Dykes of distinct composition and generation display oblique and/or perpendicular intersections.

\section{Nature and Composition}

The main petrographical and geochemical features of the dykes have been extensively discussed by many authors [16, 18, 26-29], and the relevant features are summarised here.

Petrographical parameters (mineralogical assemblage and texture) and field relations (relative chronology of events and spatial distribution) allow recognition of three compositionally types of dykes showing distinct relationship: (a) tephritic (basanites-tephrites-tephriphonolites), (b) phonolitic (phonolites-peralkaline phonolites), and (c) basaltic (alkaline basalts-trachybasalts-trachyandesitestrachytes/trachyphonolites). The first two types are geochemically grouped together forming the B-P (basanitephonolite) suite, whereas the third one into the $\mathrm{AB}-\mathrm{T}$ (alkali basalt-trachyte) suite. Mineralogical and petrographical data reported in Comin-Chiaramonti et al. $[18,26]$ and Cundari and Comin-Chiaramonti [30] show that these rocks are usually porphyritic in texture, the groundmass being finegrained or even aphyric and variable from holocrystalline to hyaline. Phenocrysts (mega and micro) are mainly represented by olivine, clinopyroxene, feldspars, (plagioclase and alkali feldspar) and feldspathoids; occasionally amphibole, biotite, garnet, and sphene can also be found. Some other general mineralogical features are: clinopyroxene composition (diopside to ferrosalite) consistently yielding $\mathrm{Al}$ enrichment trends; Fo $_{81-69}$ content of the olivine in tephrites and alkali basalts decreasing up to $65 \%$ in phonolites; zoned megacrysts of hastingsitic hornblende (core) to kaersutite (rim), associated with accessory groundmass pargasite in tephrites and phonotephrites; K-rich hastingsite and K-rich ferropargasite in phonolites; accessory groundmass mica (annite-phlogopite series), consistently yielding insufficient ( $\mathrm{Si}+\mathrm{Al}$ ) to satisfy the $\mathrm{T}$ site position; phenocryst, that is, xenocrystal plagioclase $A_{70-20}$ and $A_{74-42}$ in tephrites and phonolites, respectively; coexisting plagioclase $\left(\mathrm{An}_{14-22}\right)$ microlites associated with soda-sanidine and sanidine; feldspathoids include analcimized leucite and nepheline; Timagnetite, rarely ilmenite or hematite, as Fe-Ti oxides; and Ti-andradite and sphene as main accessory minerals.

The mineralogical assemblage of the tephritic-type includes phenocrysts of clinopyroxene ( $\left.\mathrm{Wo}_{40-50} \mathrm{Fs}_{10-19}\right)$, olivine $\left(\mathrm{Fo}_{60-85}\right)$ and leucite pseudomorphs (sanidine + nepheline) all set in a glassy groundmass consisting of microlites of clinopyroxene \pm olivine, Ti-magnetite \pm ilmenite, Ti-phlogopite-biotite, alkali feldspar $\left(\mathrm{Or}_{15-88}\right)$, and nepheline-analcime $\left(\mathrm{Ne}_{44-59} \mathrm{Ks}_{17-26}\right)$. Plagioclase (up to $A n_{74}$ ) is also present as phenocrysts in some samples. Accessory phases are amphibole (pargasite-kaersutite), apatite, and zircon. The phonolitic-type is characterized by phenocrysts of alkali feldspar $\left(\mathrm{Or}_{47-75}\right)$, leucite pseudomorphs, clinopyroxene $\left(\mathrm{Wo}_{48-50}\right)$, ferropargasite, nepheline \pm biotite \pm sphene \pm melanite (Ti-andradite up to $68 \%$ ) \pm magnetite, or hematite. Glassy groundmass has alkali feldspar, nepheline, and clinopyroxene \pm melanite \pm opaques microlites. The composition of the basaltic-type for the alkali basalts, trachybasalts, and trachyandesites shows pheno and/or microphenocrysts of clinopyroxene $\left(\mathrm{Wo}_{44-49} \mathrm{Fs}_{7-15}\right)$, olivine $\left(\mathrm{Fo}_{65-83}\right)$, plagioclase $\left(\mathrm{An}_{28-76}\right)$, magnetite, and biotite. The glassy groundmass contains microlites of clinopyroxene ( $\left.\mathrm{Wo}_{46-49} \mathrm{Fs}_{13-18}\right)$, magnetite, ilmenite, biotite, plagioclase $\left(\mathrm{An}_{20-45}\right)$, alkali feldspar $\left(\mathrm{Or}_{52-65}\right)$, nephelineanalcime $\left(\mathrm{Ne}_{37-73} \mathrm{Ks}_{22-38}\right)$, amphibole, and apatite \pm sphene \pm zircon. On the other hand, the mineralogy for the trachyphonolites and trachytes includes phenocrysts of alkali feldspar $\left(\mathrm{Or}_{60-65}\right)$, clinopyroxene $\left(\mathrm{Wo}_{46-49} \mathrm{Fs}_{14-20}\right)$, plagioclase $\left(\mathrm{An}_{14-16}\right)$, leucite pseudomorphs, amphibole, and biotite in hypocrystalline to glassy groundmass having microlites of alkali feldspar, biotite, and clinopyroxene \pm biotite \pm amphibole \pm magnetite \pm Ti-andradite \pm hematite

Geochemical analyses and Sr-Nd isotopic signatures indicate that the dykes are quite variable in composition. Gomes et al. [31] and Comin-Chiaramonti et al. [26, 28 ], using chemical data of minerals and bulk rocks and petrochemical classification, grouped all the potassic dykes into two main lines of evolution: (a) a silica-undersaturated lineage ranging from basanite to phonolite (B-P) and (b) a silica-saturated lineage ranging from alkaline basalt to trachyte (AB-T) (Figure 4), probably related to distinct parental magmas. In general, both suites show similar elemental enrichment patterns for large ion lithophile elements (LILE), that is, $\mathrm{Rb}$ and $\mathrm{Ba}$, as well as for other incompatible elements, such as $\mathrm{La}, \mathrm{Ce}, \mathrm{Sm}$, and Tb. Nevertheless, the major differences between the two groups are the higher concentrations of $\mathrm{K}_{2} \mathrm{O}, \mathrm{TiO}_{2}, \mathrm{Zr}, \mathrm{Nb}, \mathrm{Y}$, and REE of the $\mathrm{B}-\mathrm{P}$ rocks in comparison to those of the $\mathrm{AB}-\mathrm{T}$ suite. $\mathrm{Mg}$ values $\left(\mathrm{Mg}^{*}\right)$ and $\mathrm{Ni}$ and $\mathrm{Cr}$ contents indicate that all the magma-types representative of the less evolved dykes in the investigated area can be considered to some extent to be derivatives [26].

Subsequent researchers have been seeking to establish the mechanism of generation and evolution of the alkaline magmatism in Central-Eastern Paraguay. Based on textural, mineralogical and petrochemical evidence and utilizing quantitative mass balance calculations on major oxides, the latter authors $[8,18]$ concluded that fractional crystallization was a potentially important process in the formation of these rocks. Furthermore, $\mathrm{Sr}$ and $\mathrm{Nd}$ isotope data suggested that the parental magmas responsible for the alkaline potassic series (B-P and $A B-T$ ) and sodic suites occurrences, found in some areas of Eastern Paraguay (northern, central, and southern: Alto Paraguay, Asunción, and Misiones provinces, respectively (cf. [10]), would have derived from a heterogeneous subcontinental mantle lithospheric source, submitted to different melting degrees and variously enriched in 
TABLE 1: Additional information about the alkaline dykes that occur in the central segment of the Asunción Rift.

\begin{tabular}{|c|c|c|c|c|c|c|c|}
\hline Field number & Latitude/Longitude & Dip direction & Width (m) & Rock & Evolutive trend & References & Chronology \\
\hline$\overline{001}$ & $25^{\circ} 40^{\prime} 53^{\prime \prime} \mathrm{S} / 56^{\circ} 56^{\prime} 36^{\prime \prime} \mathrm{W}$ & $355 / 89$ & 1.30 & Trachybasalt & $\mathrm{AB}-\mathrm{T}$ & {$[8,21,36]$} & \\
\hline 002 & $25^{\circ} 41^{\prime} 31^{\prime \prime} \mathrm{S} / 56^{\circ} 56^{\prime} 34^{\prime \prime} \mathrm{W}$ & $342 / 89$ & 1.40 & Tephriphonolite & $\mathrm{B}-\mathrm{P}$ & This paper & \\
\hline 003 & $25^{\circ} 41^{\prime} 34^{\prime \prime} \mathrm{S} / 56^{\circ} 56^{\prime} 32^{\prime \prime} \mathrm{W}$ & $150 / 89$ & 0.40 & Phonolite & $\mathrm{B}-\mathrm{P}$ & This paper & \\
\hline 004 & $25^{\circ} 41^{\prime} 08^{\prime \prime} \mathrm{S} / 56^{\circ} 56^{\prime} 41^{\prime \prime} \mathrm{W}$ & $320 / 89$ & 1.00 & Tephriphonolite & B-P & {$[8,21,36]$} & \\
\hline 005 & $25^{\circ} 41^{\prime} 34^{\prime \prime} \mathrm{S} / 56^{\circ} 56^{\prime} 55^{\prime \prime} \mathrm{W}$ & $130 / 89$ & 0.70 & Phonolite & $\mathrm{B}-\mathrm{P}$ & This paper & \\
\hline 006 & $25^{\circ} 41^{\prime} 40^{\prime \prime} \mathrm{S} / 56^{\circ} 56^{\prime} 58^{\prime \prime} \mathrm{W}$ & $145 / 89$ & 0.90 & Phonotephrite & $\mathrm{B}-\mathrm{P}$ & This paper & \\
\hline 007 & $25^{\circ} 41^{\prime} 58^{\prime \prime} \mathrm{S} / 56^{\circ} 56^{\prime} 18^{\prime \prime} \mathrm{W}$ & $170 / 89$ & 1.70 & Phonolite & $\mathrm{B}-\mathrm{P}$ & This paper & \\
\hline 008 & $25^{\circ} 41^{\prime} 50^{\prime \prime} \mathrm{S} / 56^{\circ} 56^{\prime} 51^{\prime \prime} \mathrm{W}$ & $325 / 89$ & 1.45 & Tephrite & B-P & This paper & \\
\hline 009 & $25^{\circ} 42^{\prime} 14^{\prime \prime} \mathrm{S} / 56^{\circ} 56^{\prime} 48^{\prime \prime} \mathrm{W}$ & $310 / 89$ & 1.30 & Phonotephrite & $\mathrm{AB}-\mathrm{T}$ & This paper & \\
\hline 010 & $25^{\circ} 41^{\prime} 45^{\prime \prime} \mathrm{S} / 56^{\circ} 56^{\prime} 19^{\prime \prime} \mathrm{W}$ & $305 / 89$ & 0.45 & Trachybasalt & $\mathrm{AB}-\mathrm{T}$ & This paper & 10 is cut by 11 \\
\hline 011 & $25^{\circ} 41^{\prime} 55^{\prime \prime} \mathrm{S} / 56^{\circ} 56^{\prime} 19^{\prime \prime} \mathrm{W}$ & $130 / 89$ & 0.80 & Tephriphonolite & B-P & This paper & \\
\hline 012 & $25^{\circ} 41^{\prime} 58^{\prime \prime} \mathrm{S} / 56^{\circ} 56^{\prime} 01^{\prime \prime} \mathrm{W}$ & $320 / 89$ & 0.70 & Trachyandesite & $\mathrm{AB}-\mathrm{T}$ & {$[8,21,36]$} & \\
\hline 013 & $25^{\circ} 42^{\prime} 11^{\prime \prime} \mathrm{S} / 56^{\circ} 57^{\prime} 04^{\prime \prime} \mathrm{W}$ & $092 / 89$ & 0.60 & Peralk. Phon. & $\mathrm{B}-\mathrm{P}$ & This paper & \\
\hline 014 & $25^{\circ} 42^{\prime} 25^{\prime \prime} \mathrm{S} / 56^{\circ} 57^{\prime} 06^{\prime \prime} \mathrm{W}$ & $290 / 89$ & 1.00 & Trachyte & $\mathrm{AB}-\mathrm{T}$ & {$[8,21,36]$} & \\
\hline 015 & $25^{\circ} 42^{\prime} 39^{\prime \prime} \mathrm{S} / 56^{\circ} 57^{\prime} 09^{\prime \prime} \mathrm{W}$ & $075 / 89$ & 0.50 & Peralk. Phonol. & $\mathrm{B}-\mathrm{P}$ & {$[8,21,36]$} & \\
\hline 016 & $25^{\circ} 42^{\prime} 56^{\prime \prime} \mathrm{S} / 56^{\circ} 57^{\prime} 11^{\prime \prime} \mathrm{W}$ & $042 / 89$ & 1.20 & Tephrite & $\mathrm{B}-\mathrm{P}$ & {$[8,21,36]$} & \\
\hline 017 & $25^{\circ} 42^{\prime} 58^{\prime \prime} \mathrm{S} / 56^{\circ} 57^{\prime} 19^{\prime \prime} \mathrm{W}$ & $287 / 89$ & 0.80 & Alkali basalt & $\mathrm{AB}-\mathrm{T}$ & This paper & \\
\hline 018 & $25^{\circ} 42^{\prime} 58^{\prime \prime} \mathrm{S} / 56^{\circ} 57^{\prime} 19^{\prime \prime} \mathrm{W}$ & $020 / 89$ & 1.00 & Trachyandesite & $\mathrm{AB}-\mathrm{T}$ & This paper & \\
\hline 019 & $25^{\circ} 43^{\prime} 20^{\prime \prime} \mathrm{S} / 56^{\circ} 57^{\prime} 15^{\prime \prime} \mathrm{W}$ & $075 / 89$ & 0.50 & Phonolite & $\mathrm{B}-\mathrm{P}$ & This paper & \\
\hline 020 & $25^{\circ} 45^{\prime} 57^{\prime \prime} \mathrm{S} / 56^{\circ} 57^{\prime} 27^{\prime \prime} \mathrm{W}$ & $065 / 89$ & 0.90 & Phonolite & $\mathrm{B}-\mathrm{P}$ & This paper & \\
\hline 021 & $25^{\circ} 45^{\prime} 59^{\prime \prime} \mathrm{S} / 56^{\circ} 57^{\prime} 30^{\prime \prime} \mathrm{W}$ & $325 / 89$ & 0.35 & Trachybasalt & $\mathrm{B}-\mathrm{P}$ & {$[8,21,36]$} & \\
\hline 022 & $25^{\circ} 45^{\prime} 59^{\prime \prime} \mathrm{S} / 56^{\circ} 57^{\prime} 30^{\prime \prime} \mathrm{W}$ & $340 / 89$ & 0.20 & Phonotephrite & $\mathrm{B}-\mathrm{P}$ & This paper & \\
\hline 023 & $25^{\circ} 44^{\prime} 29^{\prime \prime} \mathrm{S} / 56^{\circ} 57^{\prime} 36^{\prime \prime} \mathrm{W}$ & $055 / 89$ & 0.70 & Tephrite & $\mathrm{B}-\mathrm{P}$ & This paper & \\
\hline 024 & $25^{\circ} 44^{\prime} 29^{\prime \prime} \mathrm{S} / 56^{\circ} 57^{\prime} 36^{\prime \prime} \mathrm{W}$ & $045 / 89$ & 0.80 & Phonotephrite & B-P & This paper & \\
\hline 025 & $25^{\circ} 39^{\prime} 45^{\prime \prime} \mathrm{S} / 56^{\circ} 58^{\prime} 11^{\prime} \mathrm{W}$ & $140 / 89$ & 1.30 & Tephriphonolite & $\mathrm{B}-\mathrm{P}$ & This paper & 25 is cut by 26 \\
\hline 026 & $25^{\circ} 39^{\prime} 50^{\prime \prime} \mathrm{S} / 56^{\circ} 58^{\prime} 13^{\prime} \mathrm{W}$ & $310 / 89$ & 0.25 & Phonolite & B-P & {$[8,21,36]$} & \\
\hline 027 & $25^{\circ} 39^{\prime} 50^{\prime \prime} \mathrm{S} / 56^{\circ} 57^{\prime} 40^{\prime} \mathrm{W}$ & $110 / 89$ & 0.20 & Trachyandesite & $\mathrm{AB}-\mathrm{T}$ & {$[8,21,36]$} & \\
\hline 028 & $25^{\circ} 40^{\prime} 06^{\prime \prime} \mathrm{S} / 56^{\circ} 57^{\prime} 44^{\prime \prime} \mathrm{W}$ & $130 / 89$ & 0.40 & Tephriphonolite & $\mathrm{B}-\mathrm{P}$ & This paper & \\
\hline 029 & $25^{\circ} 40^{\prime} 05^{\prime \prime} \mathrm{S} / 56^{\circ} 57^{\prime} 41^{\prime \prime} \mathrm{W}$ & $110 / 89$ & 0.60 & Trachybasalt & $\mathrm{B}-\mathrm{P}$ & This paper & \\
\hline 030 & $25^{\circ} 40^{\prime} 09^{\prime \prime} \mathrm{S} / 56^{\circ} 57^{\prime} 30^{\prime \prime} \mathrm{W}$ & $310 / 89$ & 0.25 & Tephrite & $\mathrm{B}-\mathrm{P}$ & This paper & \\
\hline 031 & $25^{\circ} 40^{\prime} 10^{\prime \prime} \mathrm{S} / 56^{\circ} 57^{\prime} 37^{\prime \prime} \mathrm{W}$ & $165 / 89$ & 0.35 & Peralk. Phon. & B-P & {$[8,21,36]$} & \\
\hline 032 & $25^{\circ} 40^{\prime} 17^{\prime \prime} \mathrm{S} / 56^{\circ} 57^{\prime} 42^{\prime \prime} \mathrm{W}$ & $118 / 89$ & 2.40 & Trachy & $\mathrm{AB}-\mathrm{T}$ & {$[8,21,36]$} & 32 is cut by 31 \\
\hline 033 & $25^{\circ} 40^{\prime} 27^{\prime \prime} \mathrm{S} / 56^{\circ} 57^{\prime} 40^{\prime \prime} \mathrm{W}$ & $140 / 89$ & 0.35 & Phonotephrite & B-P & {$[8,21,36]$} & \\
\hline 034 & $25^{\circ} 40^{\prime} 30^{\prime \prime} \mathrm{S} / 56^{\circ} 57^{\prime} 46^{\prime \prime} \mathrm{W}$ & $330 / 89$ & 1.20 & Tephriphonolite & $\mathrm{B}-\mathrm{P}$ & This paper & \\
\hline 035 & $25^{\circ} 40^{\prime} 32^{\prime \prime} \mathrm{S} / 56^{\circ} 57^{\prime} 43^{\prime \prime} \mathrm{W}$ & $130 / 89$ & 0.25 & Tephrite & B-P & This paper & \\
\hline 036 & $25^{\circ} 40^{\prime} 35^{\prime \prime} \mathrm{S} / 56^{\circ} 57^{\prime} 40^{\prime \prime} \mathrm{W}$ & $120 / 89$ & 0.40 & Trachyte & $\mathrm{AB}-\mathrm{T}$ & This paper & \\
\hline 037 & $25^{\circ} 40^{\prime} 37^{\prime \prime} \mathrm{S} / 56^{\circ} 57^{\prime} 39^{\prime \prime} \mathrm{W}$ & $140 / 89$ & 0.15 & Tephriphonolite & $\mathrm{B}-\mathrm{P}$ & This paper & \\
\hline 038 & $25^{\circ} 40^{\prime} 39^{\prime \prime} \mathrm{S} / 56^{\circ} 57^{\prime} 43^{\prime \prime} \mathrm{W}$ & $290 / 89$ & 1.00 & Trachyandesite & $\mathrm{AB}-\mathrm{T}$ & This paper & \\
\hline 039 & $25^{\circ} 40^{\prime} 40^{\prime \prime} \mathrm{S} / 56^{\circ} 57^{\prime} 35^{\prime \prime} \mathrm{W}$ & $310 / 89$ & 0.35 & Phonotephrite & $\mathrm{B}-\mathrm{T}$ & This paper & \\
\hline 040 & $25^{\circ} 40^{\prime} 41^{\prime \prime} \mathrm{S} / 56^{\circ} 57^{\prime} 30^{\prime \prime} \mathrm{W}$ & $165 / 89$ & 0.40 & Phonolite & $\mathrm{B}-\mathrm{P}$ & {$[8,21,36]$} & \\
\hline 041 & $25^{\circ} 40^{\prime} 40^{\prime \prime} \mathrm{S} / 56^{\circ} 57^{\prime} 50^{\prime \prime} \mathrm{W}$ & $310 / 89$ & 0.15 & Phonotephrite & B-P & {$[8,21,36]$} & \\
\hline 042 & $25^{\circ} 40^{\prime} 42^{\prime \prime} \mathrm{S} / 56^{\circ} 57^{\prime} 55^{\prime \prime} \mathrm{W}$ & $290 / 89$ & 0.25 & Trachyandesite & $\mathrm{AB}-\mathrm{T}$ & This paper & \\
\hline 043 & $25^{\circ} 40^{\prime} 53^{\prime \prime} \mathrm{S} / 56^{\circ} 57^{\prime} 32^{\prime \prime} \mathrm{W}$ & $330 / 89$ & 0.40 & Tephriphonolite & $\mathrm{B}-\mathrm{P}$ & This paper & \\
\hline 044 & $25^{\circ} 40^{\prime} 56^{\prime \prime} \mathrm{S} / 56^{\circ} 57^{\prime} 30^{\prime \prime} \mathrm{W}$ & $130 / 89$ & 0.20 & Tephriphonolite & B-P & This paper & 44 is cut by 45 \\
\hline 045 & $25^{\circ} 40^{\prime} 56^{\prime \prime} \mathrm{S} / 56^{\circ} 57^{\prime} 30^{\prime \prime} \mathrm{W}$ & $320 / 89$ & 1.50 & Peralk. Phon. & B-P & This paper & \\
\hline 046 & $25^{\circ} 41^{\prime} 02^{\prime \prime} \mathrm{S} / 56^{\circ} 57^{\prime} 40^{\prime \prime} \mathrm{W}$ & $330 / 89$ & 0.35 & Tephrite & B-P & This paper & \\
\hline 047 & $25^{\circ} 41^{\prime} 09^{\prime \prime} \mathrm{S} / 56^{\circ} 57^{\prime} 30^{\prime \prime} \mathrm{W}$ & $320 / 89$ & 0.15 & Phonolite & $\mathrm{B}-\mathrm{P}$ & This paper & \\
\hline 048 & $25^{\circ} 41^{\prime} 08^{\prime \prime} \mathrm{S} / 56^{\circ} 57^{\prime} 47^{\prime \prime} \mathrm{W}$ & $160 / 89$ & 0.40 & Phonotephrite & B-P & This paper & \\
\hline 049 & $25^{\circ} 41^{\prime} 12^{\prime \prime} \mathrm{S} / 56^{\circ} 57^{\prime} 52^{\prime \prime} \mathrm{W}$ & $145 / 89$ & 2.00 & Tephrite & $\mathrm{B}-\mathrm{P}$ & {$[8,21,36]$} & \\
\hline 050 & $25^{\circ} 41^{\prime} 17^{\prime \prime} \mathrm{S} / 56^{\circ} 57^{\prime} 42^{\prime \prime} \mathrm{W}$ & $300 / 89$ & 0.45 & Trachybasalt & $\mathrm{AB}-\mathrm{T}$ & This paper & \\
\hline
\end{tabular}


Table 1: Continued.

\begin{tabular}{|c|c|c|c|c|c|c|c|}
\hline Field number & Latitude/Longitude & Dip direction & Width (m) & Rock & Evolutive trend & References & Chronology \\
\hline 051 & $25^{\circ} 42^{\prime} 23^{\prime \prime} \mathrm{S} / 56^{\circ} 58^{\prime} 10^{\prime \prime} \mathrm{W}$ & $310 / 89$ & 0.35 & Peralk. Phon. & $\mathrm{B}-\mathrm{T}$ & {$[8,21,36]$} & \\
\hline 052 & $25^{\circ} 42^{\prime} 26^{\prime \prime} \mathrm{S} / 56^{\circ} 58^{\prime} 19^{\prime \prime} \mathrm{W}$ & $140 / 89$ & 0.90 & Tephriphonolite & B-P & This paper & \\
\hline 053 & $25^{\circ} 41^{\prime} 20^{\prime \prime} \mathrm{S} / 56^{\circ} 58^{\prime} 25^{\prime \prime} \mathrm{W}$ & $070 / 89$ & 2.30 & Phonolite & $\mathrm{B}-\mathrm{T}$ & {$[8,21,36]$} & \\
\hline 054 & $25^{\circ} 39^{\prime} 41^{\prime \prime} \mathrm{S} / 56^{\circ} 58^{\prime} 35^{\prime \prime} \mathrm{W}$ & $130 / 89$ & 0.45 & Phonotephrite & B-P & {$[8,21,36]$} & \\
\hline 055 & $25^{\circ} 39^{\prime} 42^{\prime \prime} \mathrm{S} / 56^{\circ} 58^{\prime} 30^{\prime \prime} \mathrm{W}$ & $165 / 89$ & 0.70 & Peralk. Phon. & $\mathrm{B}-\mathrm{P}$ & {$[8,21,36]$} & \\
\hline 056 & $25^{\circ} 40^{\prime} 17^{\prime \prime} \mathrm{S} / 56^{\circ} 57^{\prime} 31^{\prime \prime} \mathrm{W}$ & $130 / 89$ & 0.35 & Trachyandesite & $\mathrm{AB}-\mathrm{T}$ & This paper & \\
\hline 057 & $25^{\circ} 39^{\prime} 55^{\prime \prime} \mathrm{S} / 56^{\circ} 58^{\prime} 11^{\prime \prime} \mathrm{W}$ & $150 / 89$ & 1.00 & Tephrite & B-P & {$[8,21,36]$} & \\
\hline 058 & $25^{\circ} 39^{\prime} 35^{\prime \prime} \mathrm{S} / 56^{\circ} 58^{\prime} 36^{\prime \prime} \mathrm{W}$ & $657 / 89$ & 1.20 & Tephriphonolite & B-P & {$[8,21,36]$} & \\
\hline 059 & $25^{\circ} 40^{\prime} 37^{\prime \prime} \mathrm{S} / 56^{\circ} 58^{\prime} 34^{\prime \prime} \mathrm{W}$ & $285 / 89$ & 0.60 & Trachyte & $\mathrm{AB}-\mathrm{T}$ & {$[8,21,36]$} & \\
\hline 060 & $25^{\circ} 40^{\prime} 43^{\prime \prime} \mathrm{S} / 56^{\circ} 58^{\prime} 42^{\prime \prime} \mathrm{W}$ & $050 / 89$ & 0.80 & Trachy & $\mathrm{AB}-\mathrm{T}$ & {$[8,21,36]$} & \\
\hline 061 & $25^{\circ} 41^{\prime} 10^{\prime \prime} \mathrm{S} / 56^{\circ} 58^{\prime} 39^{\prime \prime} \mathrm{W}$ & $335 / 89$ & 1.20 & Phonotephrite & $\mathrm{B}-\mathrm{P}$ & {$[8,21,36]$} & \\
\hline 062 & $25^{\circ} 41^{\prime} 10^{\prime \prime} \mathrm{S} / 56^{\circ} 58^{\prime} 39^{\prime \prime} \mathrm{W}$ & $135 / 89$ & 1.00 & Peralk. Phon. & B-P & {$[8,21,36]$} & \\
\hline 063 & $25^{\circ} 41^{\prime} 24^{\prime \prime} \mathrm{S} / 56^{\circ} 59^{\prime} 09^{\prime \prime} \mathrm{W}$ & $285 / 89$ & 0.70 & Trachyandesite & $\mathrm{AB}-\mathrm{T}$ & {$[8,21,36]$} & 63 is cut by 62 \\
\hline 064 & $25^{\circ} 41^{\prime} 49^{\prime \prime} \mathrm{S} / 56^{\circ} 59^{\prime} 57^{\prime \prime} \mathrm{W}$ & $070 / 89$ & 1.40 & Phonolite & $\mathrm{B}-\mathrm{P}$ & {$[8,21,36]$} & \\
\hline 065 & $25^{\circ} 41^{\prime} 24^{\prime \prime} \mathrm{S} / 56^{\circ} 58^{\prime} 36^{\prime \prime} \mathrm{W}$ & $060 / 89$ & 3.50 & Tephrite & $\mathrm{B}-\mathrm{P}$ & {$[8,21,36]$} & \\
\hline 066 & $25^{\circ} 42^{\prime} 48^{\prime \prime} \mathrm{S} / 56^{\circ} 58^{\prime} 59^{\prime \prime} \mathrm{W}$ & $050 / 89$ & 2.20 & Tephrite & B-P & {$[8,21,36]$} & \\
\hline 067 & $25^{\circ} 42^{\prime} 50^{\prime \prime} \mathrm{S} / 56^{\circ} 58^{\prime} 56^{\prime \prime} \mathrm{W}$ & $315 / 89$ & 0.20 & Phonolite & B-P & This paper & \\
\hline 068 & $25^{\circ} 42^{\prime} 53^{\prime \prime} \mathrm{S} / 56^{\circ} 58^{\prime} 35^{\prime \prime} \mathrm{W}$ & $320 / 89$ & 0.15 & Trachyte & $\mathrm{AB}-\mathrm{T}$ & This paper & \\
\hline 069 & $25^{\circ} 42^{\prime} 55^{\prime \prime} \mathrm{S} / 56^{\circ} 58^{\prime} 45^{\prime \prime} \mathrm{W}$ & $330 / 89$ & 0.40 & Peralk. Phon. & B-P & {$[8,21,36]$} & \\
\hline 070 & $25^{\circ} 42^{\prime} 43^{\prime \prime} \mathrm{S} / 56^{\circ} 58^{\prime} 53^{\prime \prime} \mathrm{W}$ & $230 / 89$ & 1.00 & Tephriphonolite & B-P & {$[8,21,36]$} & \\
\hline 071 & $25^{\circ} 41^{\prime} 22^{\prime \prime} \mathrm{S} / 56^{\circ} 55^{\prime} 20^{\prime \prime} \mathrm{W}$ & $260 / 89$ & 4.00 & Phonotephrite & B-P & This paper & \\
\hline 072 & $25^{\circ} 41^{\prime} 25^{\prime \prime} \mathrm{S} / 56^{\circ} 55^{\prime} 15^{\prime \prime} \mathrm{W}$ & $130 / 89$ & 0.25 & Peralk. Phon. & $\mathrm{B}-\mathrm{T}$ & This paper & \\
\hline 073 & $25^{\circ} 41^{\prime} 36^{\prime \prime} \mathrm{S} / 56^{\circ} 55^{\prime} 10^{\prime \prime} \mathrm{W}$ & $140 / 89$ & 0.15 & Tephrite & B-P & This paper & \\
\hline 074 & $25^{\circ} 41^{\prime} 39^{\prime \prime} \mathrm{S} / 56^{\circ} 55^{\prime} 04^{\prime \prime} \mathrm{W}$ & $125 / 89$ & 3.50 & Phonolite & B-P & This paper & \\
\hline 075 & $25^{\circ} 41^{\prime} 42^{\prime \prime} \mathrm{S} / 56^{\circ} 55^{\prime} 10^{\prime \prime} \mathrm{W}$ & $330 / 89$ & 0.20 & Tephriphonolite & B-P & {$[8,21,36]$} & \\
\hline 076 & $25^{\circ} 42^{\prime} 01^{\prime \prime} \mathrm{S} / 56^{\circ} 54^{\prime} 59^{\prime \prime} \mathrm{W}$ & $340 / 89$ & 0.35 & Phonolite & B-P & {$[8,21,36]$} & \\
\hline 077 & $25^{\circ} 42^{\prime} 01^{\prime \prime} \mathrm{S} / 56^{\circ} 54^{\prime} 59^{\prime \prime} \mathrm{W}$ & $110 / 89$ & 1.40 & Trachyte & $\mathrm{AB}-\mathrm{T}$ & This paper & \\
\hline 078 & $25^{\circ} 42^{\prime} 06^{\prime \prime} \mathrm{S} / 56^{\circ} 54^{\prime} 53^{\prime \prime} \mathrm{W}$ & $330 / 89$ & 0.30 & Phonolite & B-P & This paper & \\
\hline 079 & $25^{\circ} 42^{\prime} 37^{\prime \prime} \mathrm{S} / 56^{\circ} 54^{\prime} 47^{\prime \prime} \mathrm{W}$ & $150 / 89$ & 0.40 & Tephriphonolite & B-P & This paper & \\
\hline 080 & $25^{\circ} 42^{\prime} 43^{\prime \prime} \mathrm{S} / 56^{\circ} 55^{\prime} 03^{\prime \prime} \mathrm{W}$ & $335 / 89$ & 3.20 & Tephrite & B-P & This paper & \\
\hline 081 & $25^{\circ} 42^{\prime} 06^{\prime \prime} \mathrm{S} / 56^{\circ} 52^{\prime} 26^{\prime \prime} \mathrm{W}$ & $075 / 89$ & 2.30 & Peralk. Phon. & B-P & This paper & \\
\hline 082 & $25^{\circ} 42^{\prime} 18^{\prime \prime} \mathrm{S} / 56^{\circ} 56^{\prime} 03^{\prime \prime} \mathrm{W}$ & $305 / 89$ & 0.25 & Trachyte & $\mathrm{AB}-\mathrm{T}$ & {$[8,21,36]$} & \\
\hline 083 & $25^{\circ} 42^{\prime} 20^{\prime \prime} \mathrm{S} / 56^{\circ} 52^{\prime} 35^{\prime \prime} \mathrm{W}$ & $230 / 89$ & 0.40 & Tephriphonolite & B-P & {$[8,21,36]$} & \\
\hline 084 & $25^{\circ} 42^{\prime} 21^{\prime \prime} \mathrm{S} / 56^{\circ} 52^{\prime} 37^{\prime \prime} \mathrm{W}$ & $050 / 89$ & 5.00 & Tephriphonolite & B-P & This paper & \\
\hline 085 & $25^{\circ} 42^{\prime} 40^{\prime \prime} \mathrm{S} / 56^{\circ} 53^{\prime} 29^{\prime \prime} \mathrm{W}$ & $140 / 89$ & 0.35 & Tephrite & B-P & This paper & \\
\hline 086 & $25^{\circ} 42^{\prime} 57^{\prime \prime} \mathrm{S} / 56^{\circ} 53^{\prime} 39^{\prime \prime} \mathrm{W}$ & $310 / 89$ & 0.15 & Tephrite & B-P & This paper & \\
\hline 087 & $25^{\circ} 43^{\prime} 02^{\prime \prime} \mathrm{S} / 56^{\circ} 53^{\prime} 19^{\prime \prime} \mathrm{W}$ & $065 / 89$ & 1.50 & Phonolite & B-P & This paper & \\
\hline 088 & $25^{\circ} 42^{\prime} 22^{\prime \prime} \mathrm{S} / 56^{\circ} 53^{\prime} 35^{\prime \prime} \mathrm{W}$ & $300 / 89$ & 0.30 & Tephriphonolite & B-P & This paper & \\
\hline 089 & $25^{\circ} 42^{\prime} 25^{\prime \prime} \mathrm{S} / 56^{\circ} 53^{\prime} 33^{\prime \prime} \mathrm{W}$ & $345 / 89$ & 0.25 & Phonolite & B-P & This paper & \\
\hline 090 & $25^{\circ} 42^{\prime} 29^{\prime \prime} \mathrm{S} / 56^{\circ} 53^{\prime} 39^{\prime \prime} \mathrm{W}$ & $230 / 89$ & 10.0 & Trachybasalt & $\mathrm{AB}-\mathrm{T}$ & This paper & 90 is cut by 91 \\
\hline 091 & $25^{\circ} 42^{\prime} 29^{\prime \prime} \mathrm{S} / 56^{\circ} 54^{\prime} 39^{\prime \prime} \mathrm{W}$ & $330 / 89$ & 0.35 & Tephrite & B-P & This paper & \\
\hline 092 & $25^{\circ} 42^{\prime} 40^{\prime \prime} \mathrm{S} / 56^{\circ} 54^{\prime} 12^{\prime \prime} \mathrm{W}$ & $150 / 89$ & 0.25 & Phonolite & $\mathrm{B}-\mathrm{T}$ & This paper & \\
\hline 093 & $25^{\circ} 43^{\prime} 02^{\prime \prime} \mathrm{S} / 56^{\circ} 54^{\prime} 20^{\prime \prime} \mathrm{W}$ & $110 / 89$ & 2.20 & Trachyandesite & $\mathrm{AB}-\mathrm{T}$ & This paper & \\
\hline 094 & $25^{\circ} 43^{\prime} 02^{\prime \prime} \mathrm{S} / 56^{\circ} 54^{\prime} 33^{\prime \prime} \mathrm{W}$ & $140 / 89$ & 0.15 & Phonotephrite & B-P & This paper & \\
\hline 095 & $25^{\circ} 43^{\prime} 004^{\prime \prime} \mathrm{S} / 56^{\circ} 54^{\prime} 32^{\prime \prime} \mathrm{W}$ & $320 / 89$ & 0.35 & Tephriphonolite & $\mathrm{B}-\mathrm{P}$ & {$[8,21,36]$} & \\
\hline 096 & $25^{\circ} 43^{\prime} 04^{\prime \prime} \mathrm{S} / 56^{\circ} 54^{\prime} 36^{\prime \prime} \mathrm{W}$ & $320 / 89$ & 3.00 & Phonotephrite & B-P & This paper & \\
\hline 097 & $25^{\circ} 43^{\prime} 25^{\prime \prime} \mathrm{S} / 56^{\circ} 56^{\prime} 40^{\prime \prime} \mathrm{W}$ & $300 / 89$ & 0.20 & Tephrite & B-P & {$[8,21,36]$} & \\
\hline 098 & $25^{\circ} 43^{\prime} 30^{\prime \prime} \mathrm{S} / 56^{\circ} 56^{\prime} 41^{\prime \prime} \mathrm{W}$ & $310 / 89$ & 0.35 & Tephrite & $\mathrm{B}-\mathrm{P}$ & {$[8,21,36]$} & \\
\hline 099 & $25^{\circ} 43^{\prime} 37^{\prime \prime} \mathrm{S} / 56^{\circ} 56^{\prime} 43^{\prime \prime} \mathrm{W}$ & $250 / 89$ & 0.80 & Peralk. Phon. & $\mathrm{B}-\mathrm{P}$ & This paper & \\
\hline 100 & $25^{\circ} 43^{\prime} 39^{\prime \prime} \mathrm{S} / 56^{\circ} 56^{\prime} 40^{\prime \prime} \mathrm{W}$ & $130 / 89$ & 0.35 & Phonotephrite & $\mathrm{B}-\mathrm{P}$ & This paper & \\
\hline
\end{tabular}


TABle 1: Continued.

\begin{tabular}{|c|c|c|c|c|c|c|c|}
\hline Field number & Latitude/Longitude & Dip direction & Width $(\mathrm{m})$ & Rock & Evolutive trend & References & Chronology \\
\hline 101 & $25^{\circ} 43^{\prime} 41^{\prime \prime} \mathrm{S} / 56^{\circ} 56^{\prime} 37^{\prime \prime} \mathrm{W}$ & $140 / 89$ & 0.20 & Phonotephrite & B-P & This paper & \\
\hline 102 & $25^{\circ} 43^{\prime} 59^{\prime \prime} \mathrm{S} / 56^{\circ} 54^{\prime} 29^{\prime \prime} \mathrm{W}$ & $300 / 89$ & 0.60 & Trachyphonolite & $\mathrm{AB}-\mathrm{T}$ & This paper & \\
\hline 103 & $25^{\circ} 44^{\prime} 02^{\prime \prime} \mathrm{S} / 56^{\circ} 54^{\prime} 35^{\prime \prime} \mathrm{W}$ & $125 / 89$ & 1.25 & Peralk. Phon. & $\mathrm{B}-\mathrm{P}$ & {$[8,21,36]$} & \\
\hline 104 & $25^{\circ} 43^{\prime} 13^{\prime \prime} \mathrm{S} / 56^{\circ} 54^{\prime} 44^{\prime \prime} \mathrm{W}$ & $125 / 89$ & 0.40 & Trachyphonolite & $\mathrm{AB}-\mathrm{T}$ & {$[8,21,36]$} & \\
\hline 105 & $25^{\circ} 44^{\prime} 13^{\prime \prime} \mathrm{S} / 56^{\circ} 54^{\prime} 24^{\prime \prime} \mathrm{W}$ & $120 / 89$ & 0.25 & Tephriphonolite & B-P & {$[8,21,36]$} & \\
\hline 106 & $25^{\circ} 44^{\prime} 13^{\prime \prime} \mathrm{S} / 56^{\circ} 54^{\prime} 24^{\prime \prime} \mathrm{W}$ & $155 / 89$ & 2.00 & Tephrite & B-P & This paper & \\
\hline 107 & $25^{\circ} 44^{\prime} 17^{\prime \prime} \mathrm{S} / 56^{\circ} 54^{\prime} 36^{\prime \prime} \mathrm{W}$ & $125 / 89$ & 1.80 & Trachyte & $\mathrm{AB}-\mathrm{T}$ & This paper & \\
\hline 108 & $25^{\circ} 44^{\prime} 35^{\prime \prime} \mathrm{S} / 56^{\circ} 55^{\prime} 07^{\prime \prime} \mathrm{W}$ & $345 / 89$ & 0.70 & Tephriphonolite & $\mathrm{B}-\mathrm{P}$ & This paper & \\
\hline 109 & $25^{\circ} 44^{\prime} 40^{\prime \prime} \mathrm{S} / 56^{\circ} 55^{\prime} 10^{\prime \prime} \mathrm{W}$ & $160 / 89$ & 1.00 & Tephrite & B-P & This paper & 109 is cut by 110 \\
\hline 110 & $25^{\circ} 44^{\prime} 40^{\prime \prime} \mathrm{S} / 56^{\circ} 55^{\prime} 10^{\prime \prime} \mathrm{W}$ & $345 / 89$ & 3.20 & Phonolite & B-P & This paper & \\
\hline 111 & $25^{\circ} 46^{\prime} 26^{\prime \prime} \mathrm{S} / 56^{\circ} 56^{\prime} 17^{\prime \prime} \mathrm{W}$ & $295 / 89$ & 1.60 & Tephriphonolite & B-P & This paper & \\
\hline 112 & $25^{\circ} 47^{\prime} 06^{\prime \prime} \mathrm{S} / 56^{\circ} 56^{\prime} 14^{\prime \prime} \mathrm{W}$ & $170 / 89$ & 2.60 & Peralk. Phon. & B-P & This paper & \\
\hline 113 & $25^{\circ} 47^{\prime} 39^{\prime \prime} \mathrm{S} / 56^{\circ} 56^{\prime} 18^{\prime \prime} \mathrm{W}$ & $305 / 89$ & 3.20 & Phonotephrite & B-P & This paper & \\
\hline 114 & $25^{\circ} 47^{\prime} 42^{\prime \prime} \mathrm{S} / 56^{\circ} 56^{\prime} 16^{\prime \prime} \mathrm{W}$ & $345 / 89$ & 0.55 & Phonolite & B-P & This paper & \\
\hline 115 & $25^{\circ} 47^{\prime} 44^{\prime \prime} \mathrm{S} / 56^{\circ} 56^{\prime} 18^{\prime \prime} \mathrm{W}$ & $130 / 89$ & 8.00 & Trachyphonolite & $\mathrm{AB}-\mathrm{T}$ & This paper & \\
\hline 116 & $25^{\circ} 47^{\prime} 46^{\prime \prime} \mathrm{S} / 56^{\circ} 56^{\prime} 20^{\prime \prime} \mathrm{W}$ & $155 / 89$ & 0.40 & Phonolite & B-P & {$[8,21,36]$} & \\
\hline 117 & $25^{\circ} 47^{\prime} 46^{\prime \prime} \mathrm{S} / 56^{\circ} 56^{\prime} 19^{\prime \prime} \mathrm{W}$ & $330 / 89$ & 0.25 & Tephriphonolite & B-P & {$[8,21,36]$} & \\
\hline 118 & $25^{\circ} 47^{\prime} 46^{\prime \prime} \mathrm{S} / 56^{\circ} 56^{\prime} 19^{\prime \prime} \mathrm{W}$ & $150 / 89$ & 2.50 & Phonolite & B-P & This paper & \\
\hline 119 & $25^{\circ} 48^{\prime} 04^{\prime \prime} \mathrm{S} / 56^{\circ} 56^{\prime} 22^{\prime \prime} \mathrm{W}$ & $315 / 89$ & 1.70 & Tephriphonolite & B-P & This paper & \\
\hline 120 & $25^{\circ} 48^{\prime} 27^{\prime \prime} \mathrm{S} / 56^{\circ} 56^{\prime} 0 / 5^{\prime \prime} \mathrm{W}$ & $125 / 89$ & 0.35 & Trachyandesite & $\mathrm{AB}-\mathrm{T}$ & {$[8,21,36]$} & \\
\hline 121 & $25^{\circ} 50^{\prime} 43^{\prime \prime} \mathrm{S} / 56^{\circ} 56^{\prime} 22^{\prime \prime} \mathrm{W}$ & $155 / 89$ & 1.20 & Phonolite & $\mathrm{B}-\mathrm{P}$ & This paper & \\
\hline 122 & $25^{\circ} 48^{\prime} 45^{\prime \prime} \mathrm{S} / 56^{\circ} 56^{\prime} 30^{\prime \prime} \mathrm{W}$ & $340 / 89$ & 2.70 & Phonolite & $\mathrm{B}-\mathrm{T}$ & This paper & \\
\hline 123 & $25^{\circ} 51^{\prime} 28^{\prime \prime} \mathrm{S} / 56^{\circ} 55^{\prime} 37^{\prime \prime} \mathrm{W}$ & $160 / 89$ & 0.80 & Tephrite & B-P & This paper & \\
\hline 124 & $25^{\circ} 47^{\prime} 37^{\prime \prime} \mathrm{S} / 56^{\circ} 57^{\prime} 05^{\prime \prime} \mathrm{W}$ & $160 / 89$ & 0.60 & Phonolite & B-P & This paper & \\
\hline 125 & $25^{\circ} 47^{\prime} 45^{\prime \prime} \mathrm{S} / 56^{\circ} 57^{\prime} 06^{\prime \prime} \mathrm{W}$ & $125 / 89$ & 2.40 & Tephriphonolite & $\mathrm{B}-\mathrm{P}$ & This paper & \\
\hline 126 & $25^{\circ} 47^{\prime} 45^{\prime \prime} \mathrm{S} / 56^{\circ} 57^{\prime} 04^{\prime \prime} \mathrm{W}$ & $320 / 89$ & 1.80 & Trachyandesite & $\mathrm{AB}-\mathrm{T}$ & This paper & \\
\hline 127 & $25^{\circ} 47^{\prime} 57^{\prime \prime} \mathrm{S} / 56^{\circ} 57^{\prime} 10^{\prime \prime} \mathrm{W}$ & $330 / 89$ & 1.00 & Phonotephrite & $\mathrm{B}-\mathrm{P}$ & This paper & \\
\hline 128 & $25^{\circ} 47^{\prime} 59^{\prime \prime} \mathrm{S} / 56^{\circ} 57^{\prime} 12^{\prime \prime} \mathrm{W}$ & $310 / 89$ & 0.20 & Tephrite & B-P & This paper & \\
\hline 129 & $25^{\circ} 48^{\prime} 43^{\prime \prime} \mathrm{S} / 56^{\circ} 57^{\prime} 091^{\prime \prime} \mathrm{W}$ & $315 / 89$ & 0.35 & Tephrite & B-P & This paper & \\
\hline 130 & $25^{\circ} 48^{\prime} 45^{\prime \prime} \mathrm{S} / 56^{\circ} 57^{\prime} 11^{\prime \prime} \mathrm{W}$ & $340 / 89$ & 2.70 & Peralk. Phon. & B-P & This paper & \\
\hline 131 & $25^{\circ} 48^{\prime} 45^{\prime \prime} \mathrm{S} / 56^{\circ} 57^{\prime} 11^{\prime \prime} \mathrm{W}$ & $300 / 89$ & 0.25 & Trachyphonolite & $\mathrm{AB}-\mathrm{T}$ & {$[8,21,36]$} & \\
\hline 132 & $25^{\circ} 48^{\prime} 43^{\prime \prime} \mathrm{S} / 56^{\circ} 57^{\prime} 09^{\prime \prime} \mathrm{W}$ & $150 / 89$ & 0.35 & Peralk. Phon. & $\mathrm{B}-\mathrm{P}$ & This paper & \\
\hline 133 & $25^{\circ} 49^{\prime} 53^{\prime \prime} \mathrm{S} / 56^{\circ} 57^{\prime} 43^{\prime \prime} \mathrm{W}$ & $330 / 89$ & 0.20 & Peralk. Phon. & $\mathrm{B}-\mathrm{P}$ & This paper & \\
\hline 134 & $25^{\circ} 49^{\prime} 53^{\prime \prime} \mathrm{S} / 56^{\circ} 57^{\prime} 43^{\prime \prime} \mathrm{W}$ & $030 / 89$ & 0.70 & Trachyte & $\mathrm{AB}-\mathrm{T}$ & This paper & 134 is cut by 133 \\
\hline 135 & $25^{\circ} 49^{\prime} 53^{\prime \prime} \mathrm{S} / 56^{\circ} 57^{\prime} 43^{\prime \prime} \mathrm{W}$ & $150 / 89$ & 0.35 & Phonotephrite & $\mathrm{B}-\mathrm{T}$ & {$[8,21,36]$} & \\
\hline 136 & $25^{\circ} 49^{\prime} 55^{\prime \prime} \mathrm{S} / 56^{\circ} 57^{\prime} 40^{\prime \prime} \mathrm{W}$ & $070 / 89$ & 0.20 & Phonolite & $\mathrm{B}-\mathrm{P}$ & This paper & \\
\hline 137 & $25^{\circ} 47^{\prime} 55^{\prime \prime} \mathrm{S} / 56^{\circ} 57^{\prime} 53^{\prime \prime} \mathrm{W}$ & $140 / 89$ & 0.40 & Tephrite & B-P & This paper & \\
\hline 138 & $25^{\circ} 48^{\prime} 00^{\prime \prime} \mathrm{S} / 56^{\circ} 57^{\prime} 55^{\prime \prime} \mathrm{W}$ & $300 / 89$ & 1.30 & Trachyandesite & $\mathrm{AB}-\mathrm{T}$ & This paper & \\
\hline 139 & $25^{\circ} 47^{\prime} 45^{\prime \prime} \mathrm{S} / 56^{\circ} 57^{\prime} 29^{\prime \prime} \mathrm{W}$ & $165 / 89$ & 3.00 & Tephriphonolite & B-P & This paper & \\
\hline 140 & $25^{\circ} 47^{\prime} 25^{\prime \prime} \mathrm{S} / 56^{\circ} 57^{\prime} 39^{\prime \prime} \mathrm{W}$ & $135 / 89$ & 0.70 & Trachyphonolite & $\mathrm{AB}-\mathrm{T}$ & This paper & \\
\hline 141 & $25^{\circ} 47^{\prime} 30^{\prime \prime} \mathrm{S} / 56^{\circ} 57^{\prime} 20^{\prime \prime} \mathrm{W}$ & $155 / 89$ & 0.35 & Peralk. Phon. & B-P & This paper & \\
\hline 142 & $25^{\circ} 47^{\prime} 32^{\prime \prime} \mathrm{S} / 56^{\circ} 57^{\prime} 30^{\prime \prime} \mathrm{W}$ & $31 / 89$ & 0.35 & Trachyandesite & $\mathrm{AB}-\mathrm{T}$ & {$[8,21,36]$} & \\
\hline 143 & $25^{\circ} 47^{\prime} 32^{\prime \prime} \mathrm{S} / 56^{\circ} 57^{\prime} 28^{\prime \prime} \mathrm{W}$ & $165 / 89$ & 1.50 & Phonolite & B-P & This paper & \\
\hline 144 & $25^{\circ} 47^{\prime} 30^{\prime \prime} \mathrm{S} / 56^{\circ} 57^{\prime} 25^{\prime \prime} \mathrm{W}$ & $335 / 89$ & 0.25 & Tephrite & $\mathrm{B}-\mathrm{P}$ & This paper & \\
\hline 145 & $25^{\circ} 47^{\prime} 27^{\prime \prime} \mathrm{S} / 56^{\circ} 57^{\prime} 26^{\prime \prime} \mathrm{W}$ & $115 / 89$ & 1.60 & Trachyte & $\mathrm{AB}-\mathrm{T}$ & This paper & \\
\hline 146 & $25^{\circ} 47^{\prime} 27^{\prime \prime} \mathrm{S} / 56^{\circ} 57^{\prime} 26^{\prime \prime} \mathrm{W}$ & $120 / 89$ & 0.35 & Tephriphonolite & B-P & {$[8,21,36]$} & \\
\hline 147 & $25^{\circ} 47^{\prime} 19^{\prime \prime} \mathrm{S} / 56^{\circ} 57^{\prime} 35^{\prime \prime} \mathrm{W}$ & $160 / 89$ & 0.35 & Phonolite & $\mathrm{AB}-\mathrm{T}$ & This paper & \\
\hline 148 & $25^{\circ} 47^{\prime} 29^{\prime \prime} \mathrm{S} / 56^{\circ} 57^{\prime} 30^{\prime \prime} \mathrm{W}$ & $305 / 89$ & 0.25 & Trachyphonolite & $\mathrm{AB}-\mathrm{T}$ & This paper & \\
\hline 149 & $25^{\circ} 47^{\prime} 30^{\prime \prime} \mathrm{S} / 56^{\circ} 57^{\prime} 25^{\prime \prime} \mathrm{W}$ & $330 / 89$ & 0.40 & Tephrite & $\mathrm{B}-\mathrm{P}$ & {$[8,21,36]$} & \\
\hline 150 & $25^{\circ} 47^{\prime} 35^{\prime \prime} \mathrm{S} / 56^{\circ} 57^{\prime} 23^{\prime \prime} \mathrm{W}$ & $345 / 89$ & 2.30 & Phonolite & B-P & This paper & \\
\hline
\end{tabular}


Table 1: Continued.

\begin{tabular}{|c|c|c|c|c|c|c|c|}
\hline Field number & Latitude/Longitude & Dip direction & Width $(\mathrm{m})$ & Rock & Evolutive trend & References & Chronology \\
\hline 151 & $25^{\circ} 43^{\prime} 37^{\prime \prime} \mathrm{S} / 56^{\circ} 53^{\prime} 19^{\prime \prime} \mathrm{W}$ & $240 / 89$ & 0.80 & Phonolite & B-P & This paper & \\
\hline 152 & $25^{\circ} 44^{\prime} 26^{\prime \prime} \mathrm{S} / 56^{\circ} 51^{\prime} 17^{\prime \prime} \mathrm{W}$ & $135 / 89$ & 10.0 & Phonolite & B-P & {$[8,21,36]$} & \\
\hline 153 & $25^{\circ} 44^{\prime} 28^{\prime \prime} \mathrm{S} / 56^{\circ} 51^{\prime} 30^{\prime \prime} \mathrm{W}$ & $295 / 89$ & 0.80 & Trachyte & $\mathrm{AB}-\mathrm{T}$ & {$[8,21,36]$} & \\
\hline 154 & $25^{\circ} 44^{\prime} 30^{\prime \prime} \mathrm{S} / 56^{\circ} 51^{\prime} 25^{\prime \prime} \mathrm{W}$ & $330 / 89$ & 0.45 & Peralk. Phon. & B-P & This paper & \\
\hline 155 & $25^{\circ} 44^{\prime} 07^{\prime \prime} \mathrm{S} / 56^{\circ} 52^{\prime} 08^{\prime \prime} \mathrm{W}$ & $120 / 89$ & 3.00 & Phonotephrite & B-P & This paper & \\
\hline 156 & $25^{\circ} 44^{\prime} 09^{\prime \prime} \mathrm{S} / 56^{\circ} 52^{\prime} 10^{\prime \prime} \mathrm{W}$ & $310 / 89$ & 2.70 & Tephriphonolite & B-P & This paper & \\
\hline 157 & $25^{\circ} 44^{\prime} 11^{\prime \prime} \mathrm{S} / 56^{\circ} 52^{\prime} 12^{\prime \prime} \mathrm{W}$ & $335 / 89$ & 1.20 & Peralk. Phon. & B-P & This paper & \\
\hline 158 & $25^{\circ} 44^{\prime} 09^{\prime \prime} \mathrm{S} / 56^{\circ} 52^{\prime} 23^{\prime \prime} \mathrm{W}$ & $340 / 89$ & 0.30 & Peralk. Phon. & B-P & {$[8,21,36]$} & \\
\hline 159 & $25^{\circ} 44^{\prime} 10^{\prime \prime} \mathrm{S} / 56^{\circ} 52^{\prime} 20^{\prime \prime} \mathrm{W}$ & $330 / 89$ & 0.20 & Phonotephrite & B-P & {$[8,21,36]$} & \\
\hline 160 & $25^{\circ} 44^{\prime} 15^{\prime \prime} \mathrm{S} / 56^{\circ} 52^{\prime} 15^{\prime \prime} \mathrm{W}$ & $115 / 89$ & 0.45 & Trachyphonolite & $\mathrm{AB}-\mathrm{T}$ & {$[8,21,36]$} & \\
\hline 161 & $25^{\circ} 44^{\prime} 16^{\prime \prime} \mathrm{S} / 56^{\circ} 52^{\prime} 18^{\prime \prime} \mathrm{W}$ & $130 / 89$ & 0.20 & Phonotephrite & B-P & {$[8,21,36]$} & \\
\hline 162 & $25^{\circ} 44^{\prime} 17^{\prime \prime} \mathrm{S} / 56^{\circ} 52^{\prime} 20^{\prime \prime} \mathrm{W}$ & $155 / 89$ & 0.35 & Phonolite & B-P & This paper & \\
\hline 163 & $25^{\circ} 45^{\prime} 17^{\prime \prime} \mathrm{S} / 56^{\circ} 52^{\prime} 05^{\prime \prime} \mathrm{W}$ & $320 / 89$ & 0.20 & Tephrite & B-P & This paper & \\
\hline 164 & $25^{\circ} 46^{\prime} 01^{\prime \prime} \mathrm{S} / 56^{\circ} 52^{\prime} 18^{\prime \prime} \mathrm{W}$ & $320 / 89$ & 0.60 & Phonolite & $\mathrm{B}-\mathrm{P}$ & This paper & \\
\hline 165 & $25^{\circ} 46^{\prime} 01^{\prime \prime} \mathrm{S} / 56^{\circ} 52^{\prime} 18^{\prime \prime} \mathrm{W}$ & $115 / 89$ & 1.00 & Tephrite & $\mathrm{B}-\mathrm{P}$ & This paper & 165 is cut by 164 \\
\hline 166 & $25^{\circ} 46^{\prime} 14^{\prime \prime} \mathrm{S} / 56^{\circ} 52^{\prime} 25^{\prime \prime} \mathrm{W}$ & $125 / 89$ & 2.30 & Tephrite & B-P & This paper & \\
\hline 167 & $25^{\circ} 46^{\prime} 18^{\prime \prime} \mathrm{S} / 56^{\circ} 52^{\prime} 19^{\prime \prime} \mathrm{W}$ & $330 / 89$ & 0.90 & Peralk. Phon. & B-P & This paper & \\
\hline 168 & $25^{\circ} 46^{\prime} 20^{\prime \prime} \mathrm{S} / 56^{\circ} 52^{\prime} 23^{\prime \prime} \mathrm{W}$ & $320 / 89$ & 0.40 & Tephrite & B-P & {$[8,21,36]$} & \\
\hline 169 & $25^{\circ} 46^{\prime} 23^{\prime \prime} \mathrm{S} / 56^{\circ} 52^{\prime} 36^{\prime \prime} \mathrm{W}$ & $120 / 89$ & 0.30 & Trachyte & $\mathrm{AB}-\mathrm{T}$ & {$[8,21,36]$} & \\
\hline 170 & $25^{\circ} 46^{\prime} 25^{\prime \prime} \mathrm{S} / 56^{\circ} 52^{\prime} 34^{\prime \prime} \mathrm{W}$ & $340 / 89$ & 0.40 & Phonolite & $\mathrm{B}-\mathrm{P}$ & {$[8,21,36]$} & \\
\hline 171 & $25^{\circ} 46^{\prime} 55^{\prime \prime} \mathrm{S} / 56^{\circ} 52^{\prime} 50^{\prime \prime} \mathrm{W}$ & $320 / 89$ & 0.25 & Tephriphonolite & B-P & {$[8,21,36]$} & \\
\hline 172 & $25^{\circ} 46^{\prime} 59^{\prime \prime} \mathrm{S} / 56^{\circ} 52^{\prime} 53^{\prime \prime} \mathrm{W}$ & $165 / 89$ & 1.30 & Phonolite & $\mathrm{AB}-\mathrm{T}$ & This paper & \\
\hline 173 & $25^{\circ} 47^{\prime} 05^{\prime \prime} \mathrm{S} / 56^{\circ} 52^{\prime} 50^{\prime \prime} \mathrm{W}$ & $340 / 89$ & 0.35 & Phonotephrite & B-P & This paper & \\
\hline 174 & $25^{\circ} 46^{\prime} 47^{\prime \prime} \mathrm{S} / 56^{\circ} 53^{\prime} 16^{\prime \prime} \mathrm{W}$ & $295 / 89$ & 2.40 & Trachyphonolite & $\mathrm{AB}-\mathrm{T}$ & This paper & \\
\hline 175 & $25^{\circ} 47^{\prime} 04^{\prime \prime} \mathrm{S} / 56^{\circ} 53^{\prime} 03^{\prime \prime} \mathrm{W}$ & $145 / 89$ & 1.50 & Tephriphonolite & $\mathrm{B}-\mathrm{P}$ & This paper & \\
\hline 176 & $25^{\circ} 47^{\prime} 10^{\prime \prime} \mathrm{S} / 56^{\circ} 53^{\prime} 09^{\prime \prime} \mathrm{W}$ & $170 / 89$ & 0.20 & Peralk. Phon. & B-P & This paper & \\
\hline 178 & $25^{\circ} 47^{\prime} 14^{\prime \prime} \mathrm{S} / 56^{\circ} 54^{\prime} 01^{\prime \prime} \mathrm{W}$ & $125 / 89$ & 0.30 & Trachyte & $\mathrm{AB}-\mathrm{T}$ & {$[8,21,36]$} & \\
\hline 179 & $25^{\circ} 46^{\prime} 47^{\prime \prime} \mathrm{S} / 56^{\circ} 53^{\prime} 20^{\prime \prime} \mathrm{W}$ & $330 / 89$ & 0.45 & Tephrite & B-P & {$[8,21,36]$} & \\
\hline 180 & $25^{\circ} 46^{\prime} 49^{\prime \prime} \mathrm{S} / 56^{\circ} 53^{\prime} 28^{\prime \prime} \mathrm{W}$ & $345 / 89$ & 2.20 & Phonolite & B-P & This paper & \\
\hline 181 & $25^{\circ} 46^{\prime} 44^{\prime \prime} \mathrm{S} / 56^{\circ} 53^{\prime} 47^{\prime \prime} \mathrm{W}$ & $325 / 89$ & 0.50 & Tephrite & B-P & This paper & \\
\hline 182 & $25^{\circ} 46^{\prime} 44^{\prime \prime} \mathrm{S} / 56^{\circ} 53^{\prime} 47^{\prime \prime} \mathrm{W}$ & $110 / 89$ & 1.20 & Trachyandesite & $\mathrm{AB}-\mathrm{T}$ & This paper & 182 is cut by 181 \\
\hline 183 & $25^{\circ} 45^{\prime} 41^{\prime \prime} \mathrm{S} / 56^{\circ} 48^{\prime} 21^{\prime \prime} \mathrm{W}$ & $145 / 89$ & 2.40 & Peralk. Phon. & B-P & This paper & \\
\hline 184 & $25^{\circ} 48^{\prime} 56^{\prime \prime} \mathrm{S} / 56^{\circ} 47^{\prime} 25^{\prime \prime} \mathrm{W}$ & $075 / 89$ & 0.80 & Phonolite & B-P & This paper & \\
\hline 185 & $25^{\circ} 48^{\prime} 29^{\prime \prime} \mathrm{S} / 56^{\circ} 48^{\prime} 54^{\prime \prime} \mathrm{W}$ & $340 / 89$ & 2.70 & Tephriphonolite & B-P & This paper & \\
\hline 186 & $25^{\circ} 48^{\prime} 53^{\prime \prime} \mathrm{S} / 56^{\circ} 47^{\prime} 05^{\prime \prime} \mathrm{W}$ & $135 / 89$ & 0.25 & Trachyandesite & $\mathrm{AB}-\mathrm{T}$ & {$[8,21,36]$} & \\
\hline 187 & $25^{\circ} 48^{\prime} 55^{\prime \prime} \mathrm{S} / 56^{\circ} 47^{\prime} 10^{\prime \prime} \mathrm{W}$ & $150 / 89$ & 0.40 & Tephrite & B-P & {$[8,21,36]$} & \\
\hline 188 & $25^{\circ} 48^{\prime} 58^{\prime \prime} \mathrm{S} / 56^{\circ} 47^{\prime} 15^{\prime \prime} \mathrm{W}$ & $070 / 89$ & 0.40 & Phonolite & B-P & This paper & \\
\hline 189 & $25^{\circ} 49^{\prime} 10^{\prime \prime} \mathrm{S} / 56^{\circ} 48^{\prime} 50^{\prime \prime} \mathrm{W}$ & $130 / 89$ & 0.30 & Tephriphonolite & B-P & {$[8,21,36]$} & \\
\hline 190 & $25^{\circ} 48^{\prime} 29^{\prime \prime} \mathrm{S} / 56^{\circ} 48^{\prime} 54^{\prime \prime} \mathrm{W}$ & $115 / 89$ & 1.80 & Trachyte & $\mathrm{AB}-\mathrm{T}$ & This paper & \\
\hline 191 & $25^{\circ} 48^{\prime} 32^{\prime \prime} \mathrm{S} / 56^{\circ} 52^{\prime} 52^{\prime \prime} \mathrm{W}$ & $120 / 89$ & 0.35 & Tephrite & $\mathrm{B}-\mathrm{P}$ & This paper & \\
\hline 192 & $25^{\circ} 48^{\prime} 49^{\prime \prime} \mathrm{S} / 56^{\circ} 52^{\prime} 50^{\prime \prime} \mathrm{W}$ & $130 / 89$ & 0.40 & Phonolite & $\mathrm{B}-\mathrm{T}$ & This paper & \\
\hline 193 & $25^{\circ} 48^{\prime} 59^{\prime \prime} \mathrm{S} / 56^{\circ} 52^{\prime} 55^{\prime \prime} \mathrm{W}$ & $280 / 89$ & 1.30 & Trachyphonolite & $\mathrm{AB}-\mathrm{T}$ & This paper & \\
\hline 194 & $25^{\circ} 49^{\prime} 02^{\prime \prime} \mathrm{S} / 56^{\circ} 52^{\prime} 50^{\prime \prime} \mathrm{W}$ & $150 / 89$ & 0.35 & Phonolite & $\mathrm{B}-\mathrm{P}$ & This paper & \\
\hline 195 & $25^{\circ} 49^{\prime} 15^{\prime \prime} \mathrm{S} / 56^{\circ} 51^{\prime} 20^{\prime \prime} \mathrm{W}$ & $320 / 89$ & 0.20 & Phonotephrite & B-P & {$[8,21,36]$} & \\
\hline 196 & $25^{\circ} 49^{\prime} 18^{\prime \prime} \mathrm{S} / 56^{\circ} 51^{\prime} 15^{\prime \prime} \mathrm{W}$ & $140 / 89$ & 0.80 & Phonolite & $\mathrm{B}-\mathrm{P}$ & This paper & \\
\hline 197 & $25^{\circ} 49^{\prime} 14^{\prime \prime} \mathrm{S} / 56^{\circ} 53^{\prime} 09^{\prime \prime} \mathrm{W}$ & $330 / 89$ & 3.00 & Tephrite & $\mathrm{B}-\mathrm{P}$ & This paper & \\
\hline 198 & $25^{\circ} 49^{\prime} 10^{\prime \prime} \mathrm{S} / 56^{\circ} 53^{\prime} 22^{\prime \prime} \mathrm{W}$ & $320 / 89$ & 0.20 & Phonolite & B-P & {$[8,21,36]$} & \\
\hline 199 & $25^{\circ} 48^{\prime} 40^{\prime \prime} \mathrm{S} / 56^{\circ} 48^{\prime} 32^{\prime \prime} \mathrm{W}$ & $315 / 89$ & 0.40 & Trachyandesite & $\mathrm{AB}-\mathrm{T}$ & {$[8,21,36]$} & \\
\hline 200 & $25^{\circ} 49^{\prime} 37^{\prime \prime} \mathrm{S} / 56^{\circ} 53^{\prime} 24^{\prime \prime} \mathrm{W}$ & $160 / 89$ & 0.25 & Peralk. Phon. & $\mathrm{B}-\mathrm{P}$ & This paper & \\
\hline
\end{tabular}


TABle 1: Continued.

\begin{tabular}{lcccccc}
\hline Field number & Latitude/Longitude & Dip direction & Width $(\mathrm{m})$ & Rock & Evolutive trend & References \\
\hline 201 & $25^{\circ} 49^{\prime} 38^{\prime \prime} \mathrm{S} / 56^{\circ} 53^{\prime} 04^{\prime \prime} \mathrm{W}$ & $310 / 89$ & 1.80 & Trachyphonolite & AB-T & This paper \\
202 & $25^{\circ} 48^{\prime} 09^{\prime \prime} \mathrm{S} / 56^{\circ} 59^{\prime} 10^{\prime \prime} \mathrm{W}$ & $165 / 89$ & 1.50 & Peralk. Phon. & AB-T & This paper \\
203 & $25^{\circ} 50^{\prime} 40^{\prime \prime} \mathrm{S} / 56^{\circ} 52^{\prime} 47^{\prime \prime} \mathrm{W}$ & $075 / 89$ & 0.60 & Phonolite & B-P & This paper \\
204 & $25^{\circ} 48^{\prime} 46^{\prime \prime} \mathrm{S} / 56^{\circ} 52^{\prime} 31^{\prime \prime} \mathrm{W}$ & $145 / 89$ & 1.00 & Tephriphonolite & B-P & This paper \\
205 & $25^{\circ} 47^{\prime} 46^{\prime \prime} \mathrm{S} / 56^{\circ} 52^{\prime} 43^{\prime \prime} \mathrm{W}$ & $230 / 89$ & 2.10 & Phonotephrite & B-P & This paper \\
206 & $25^{\circ} 47^{\prime} 45^{\prime \prime} \mathrm{S} / 56^{\circ} 52^{\prime} 40^{\prime \prime} \mathrm{W}$ & $070 / 89$ & 0.80 & Tephrite & B-P & This paper \\
\hline
\end{tabular}

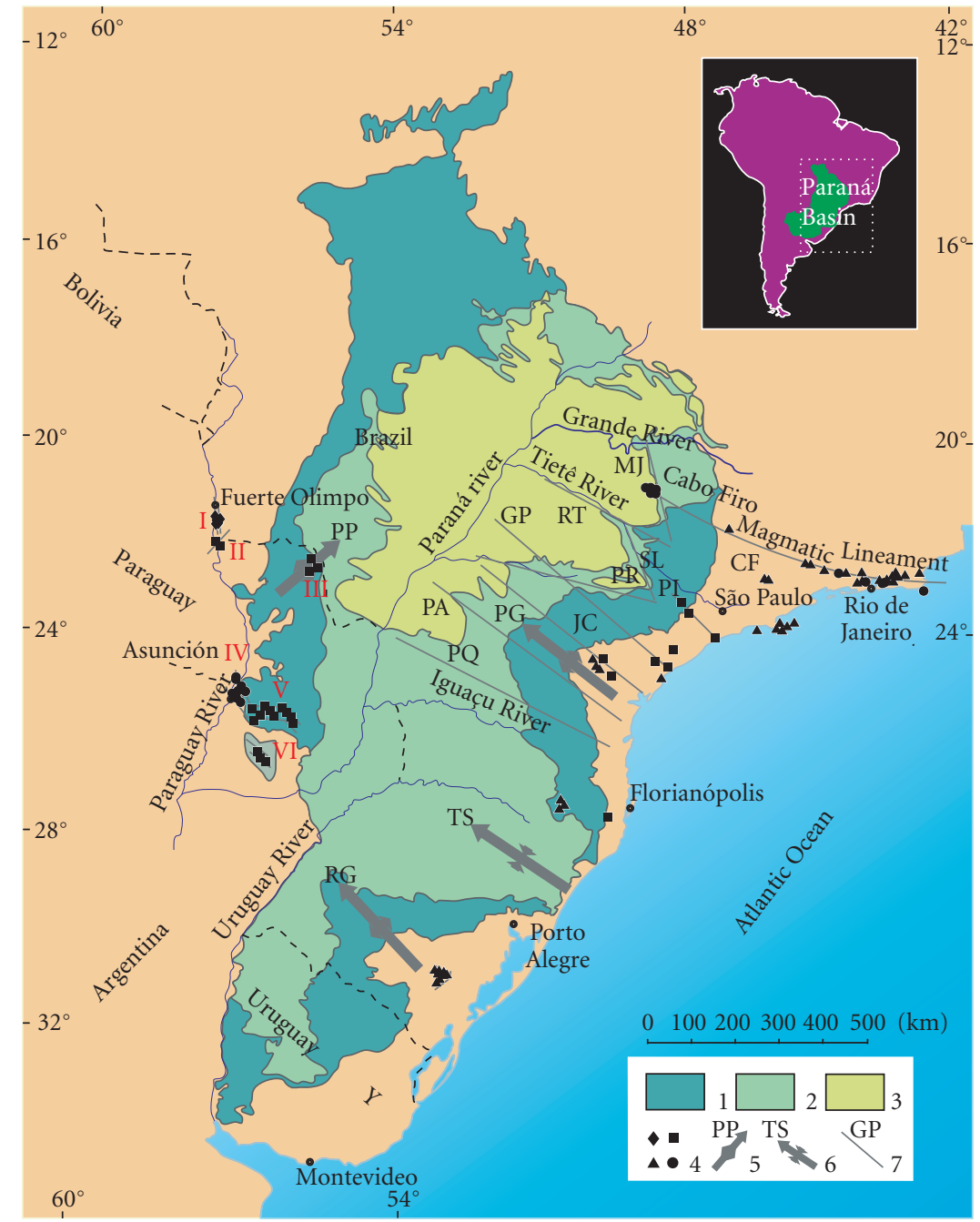

FIGURE 1: Occurrence of the alkaline intrusions in the southeastern region of the Brazilian Platform and their relationships with major structural features after Riccomini et al. [60]. (1) Late Ordovician to Early Cretaceous Paraná Basin, (2) Early Cretaceous tholeiitic lavaflows, (3) Late Cretaceous Bauru Basin, (4) Age of the alkaline intrusions (diamonds, Permian-Triassic; squares, Early Cretaceous; triangles, Late Cretaceous; circles, Paleogene), (5) Axes of main archs (PG, Ponta Grossa; RG, Rio Grande; PP, Ponta Porã), (6) Torres Syncline, and (7) Major fracture zones (MJ, Moji-Guaçu; CF, Cabo Frio; RT, Rio Tietê; SL, São Carlos-Leme; PR, Paranapanema; PI, Piedade; GP, Guapiara; RA, Rio Alonzo; PQ, Rio Piquiri). Alkaline provinces locations in Paraguay are as follows: I Alto Paraguay; II Rio Apa; III Amambay; IV Asunción; V Central; VI Misiones.

incompatible elements during Proterozoic times. Significant $\mathrm{H}_{2} \mathrm{O}, \mathrm{CO}_{2}$, and $\mathrm{F}$ are expected in the mantle source (s) considering the occurrence of coeval carbonatites $[7,18$, 32]. Comin-Chiaramonti et al. [26] also suggested that the complex compositional variation registered in the dykes of the Central Province would have resulted from multiple reequilibrium of the crystallized phases at shallow levels, in a volcanic pressure regime. 

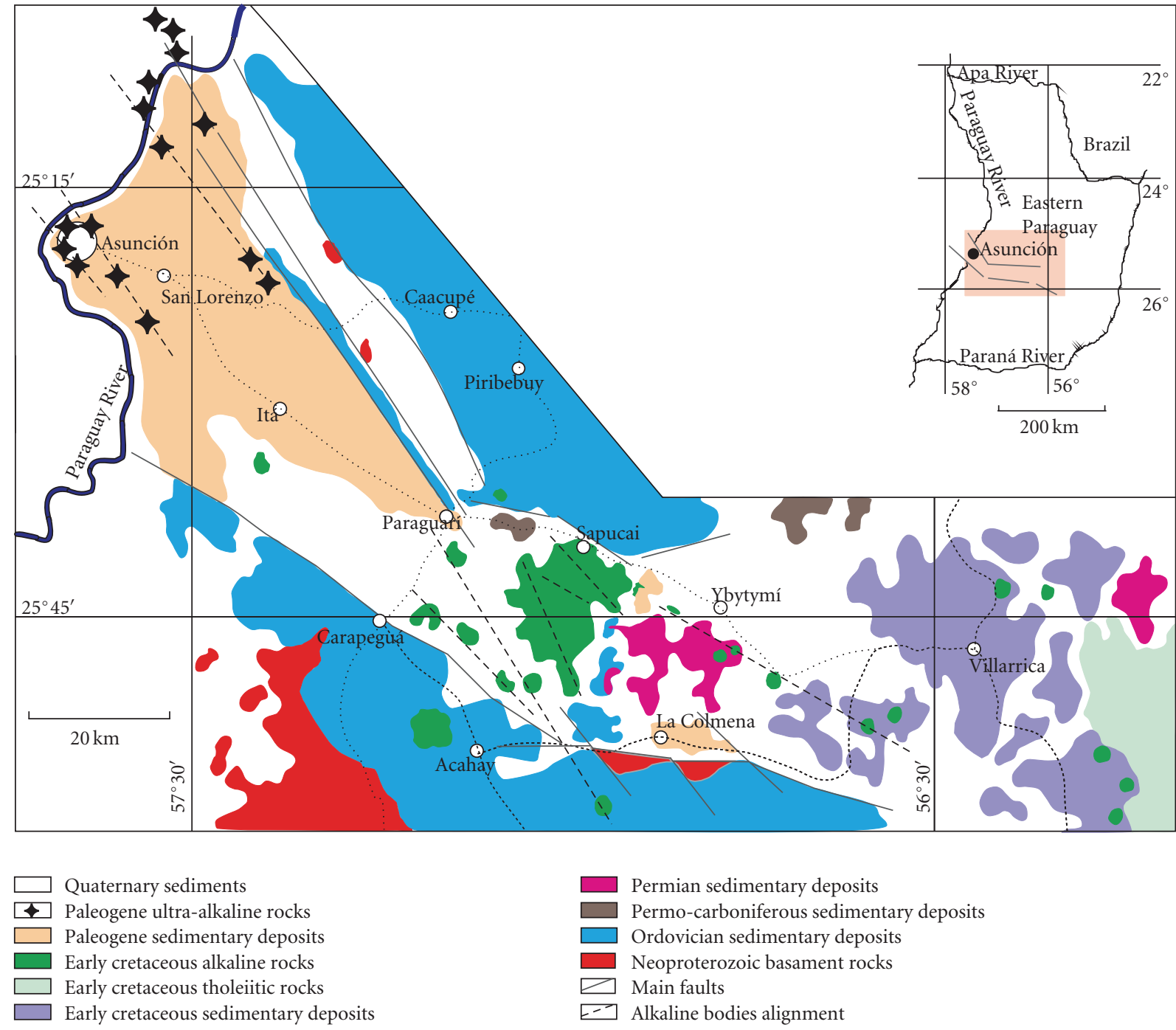

FIGURE 2: Geological map of the Asunción Rift and associated alkaline occurrences (after Velázquez et al. [17]).

\section{Emplacement Ages}

The dykes occur associated spatially and temporally with the alkaline lavas, stocks, and plugs. In order to better separate these events, a critical review of the geochronological and paleomagnetic data available in the literature for the dykes will be done following.

The K/Ar whole rock and mineral radiometric ages on a few dykes from the Sapucai area are reported in Velázquez et al. [33] and Gomes et al. [16]. Similar to the data listed in Palmieri [34] and Bitschene [23], these ages display a peak interval between 130-125 Ma. On the other hand, $\mathrm{Rb} / \mathrm{Sr}$ internal isochron for some intrusive bodies reveal ages of $128 \pm 8 \mathrm{Ma}$ and $127 \pm 7 \mathrm{Ma}[23,33] .{ }^{40} \mathrm{Ar} /{ }^{39} \mathrm{Ar}$ ages determined on phlogopite separates from Sapucai-Villarrica lamprophyre dykes indicate an average value of 127.5 Ma [7].

More recently, a detailed geochronological work using the ${ }^{40} \mathrm{Ar} /{ }^{39} \mathrm{Ar}$ method was performed by Gomes et al. [35] and Comin-Chiaramonti et al. [10]. The complete set of radiometric data obtained on biotite and plagioclase separates, as well as whole rocks samples, indicate that the dykes and some early alkaline intrusions (stocks and plugs) occur within a time interval of 128-126 Ma, with the latter authors proposing an average value of $126.4 \pm 0.4 \mathrm{Ma}$ for the alkaline potassic magmatism of the Central Province.

An extensive paleomagnetic study on the alkaline rocks and associated dykes of the Sapucai region was driven by Ernesto et al. [36]. According to those authors, the dykes acquired two opposite polarities of primary magnetization, normal and reversed, during the emplacement. The crosscutting intrusion relationships between the dykes indicate that the normal primary magnetizations are younger than reversed.

Although the geochronological data available in the literature for the alkaline potassic magmatism of the Central Province point to wide interval (130-125 Ma), highprecision ${ }^{40} \mathrm{Ar} /{ }^{39} \mathrm{Ar}$ ages indicate a short period of time (126-127 Ma, cf. [7, 10]). The field evidence suggests that the event would have occurred in a successive way, through 


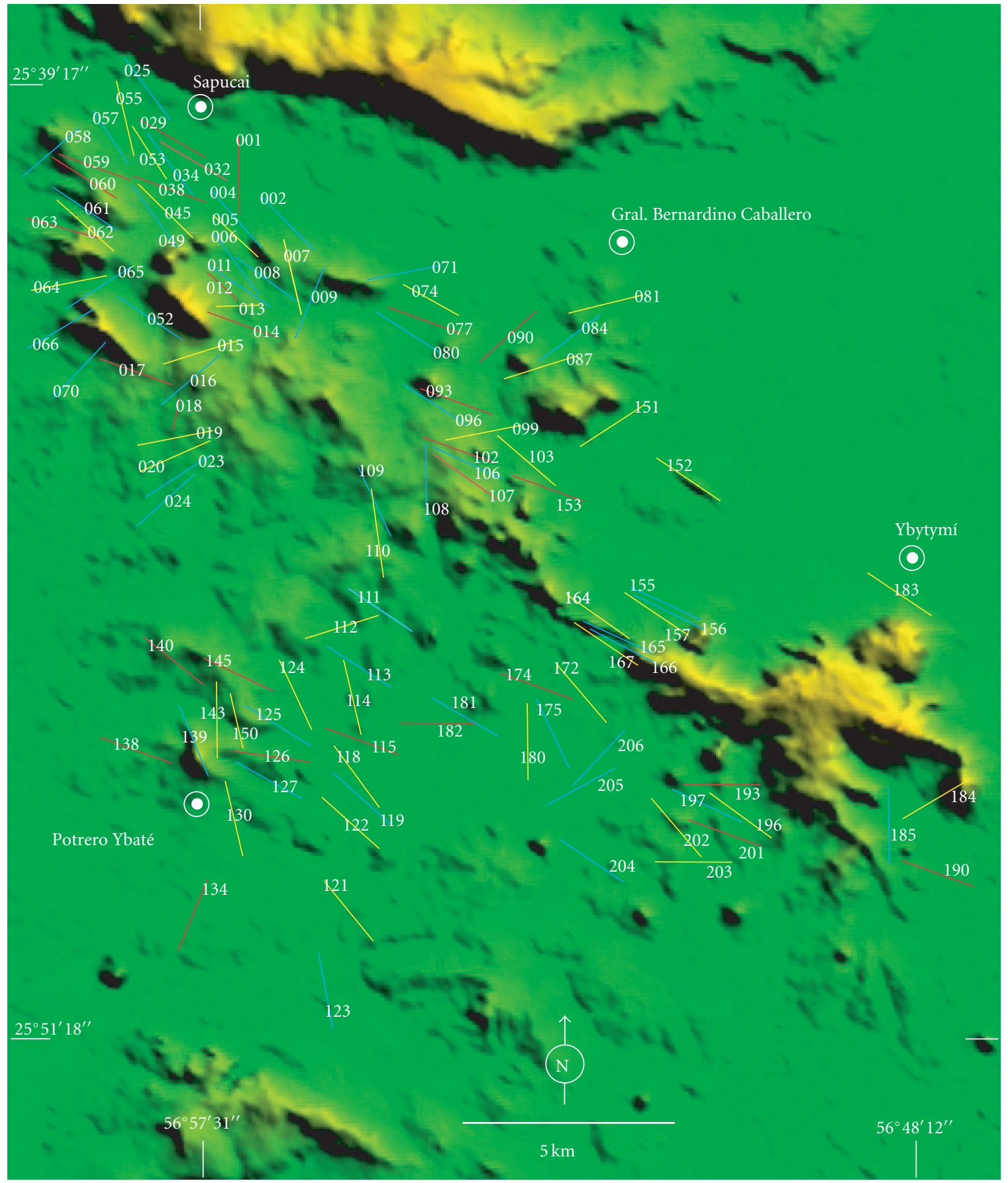

FIgURE 3: Location map of the alkaline dykes that occur in the central segment of the Asunción Rift. Additional related information is available in Table 1. Dykes with width less than $50 \mathrm{~cm}$ are not represented here.

a series of magmatic pulses. The lavas and other major intrusions (ring complexes, plugs, and stocks) predate the dyke intrusions. This fact increases the possibility that all the dyke swarm was formed in a short period of time (less than $1 \mathrm{Ma}$ ) and, mainly, during two opposite polarities of primary magnetization, as suggested by the available paleomagnetic data (Figure 5).

\section{Orientation of the Dykes and Their Relation with the Regional Structures}

Figure 6 shows the orientation of all the dykes measured in the central segment of the Asunción Rift. The measurements refer to dykes with intrusive contacts, showing clear chilled margins in $90 \%$ of cases. Two preferential orientations are recognized, NW-SE and NE-SW, the first being more 


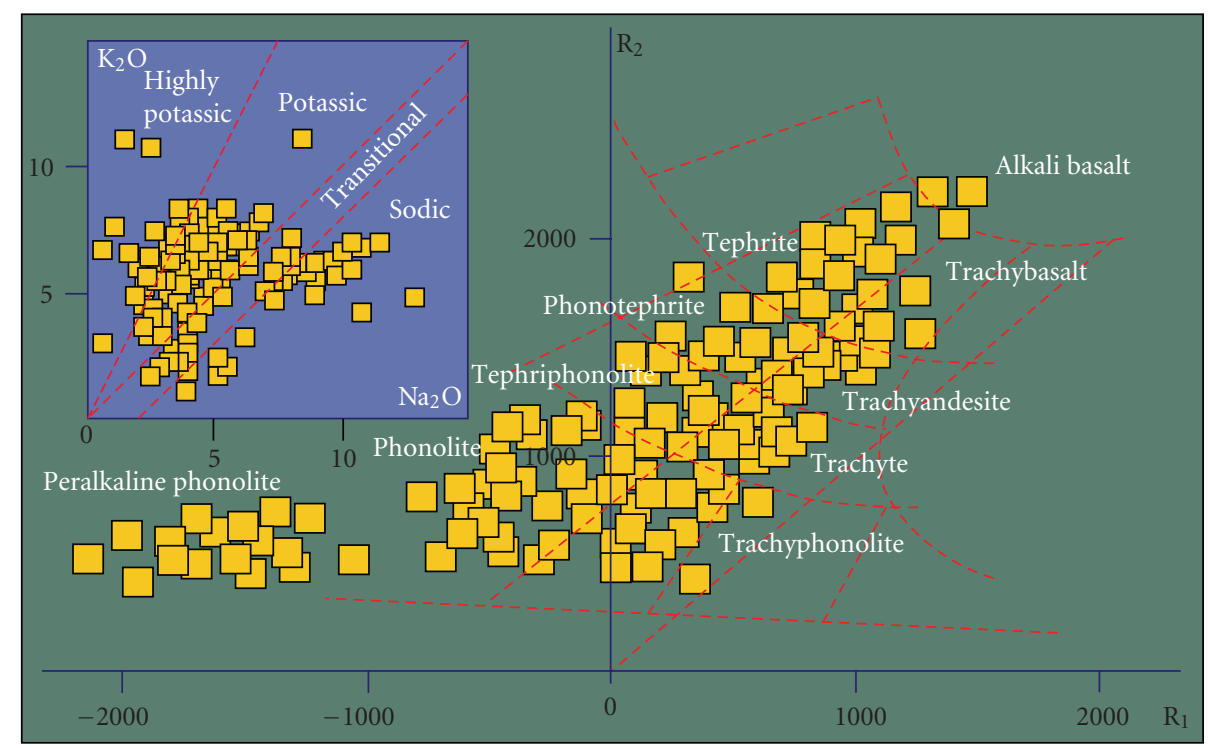

Figure 4: Compositional variation in the diagram $\mathrm{R}_{1}(4 \mathrm{Si}-11 \mathrm{Na}+\mathrm{K}-2 \mathrm{Fe}+\mathrm{Ti})-\mathrm{R}_{2}(6 \mathrm{Ca}+2 \mathrm{Mg}+2 \mathrm{Al})$ (De La Roche et al. [64]) for the alkaline dykes that occur in the central segment of the Asunción Rift. Inset: $\mathrm{K}_{2} \mathrm{O}$ versus $\mathrm{Na}_{2} \mathrm{O}$ diagram showing the transition between the two series (fields according to [18]). Data source: $[8,26,31]$.
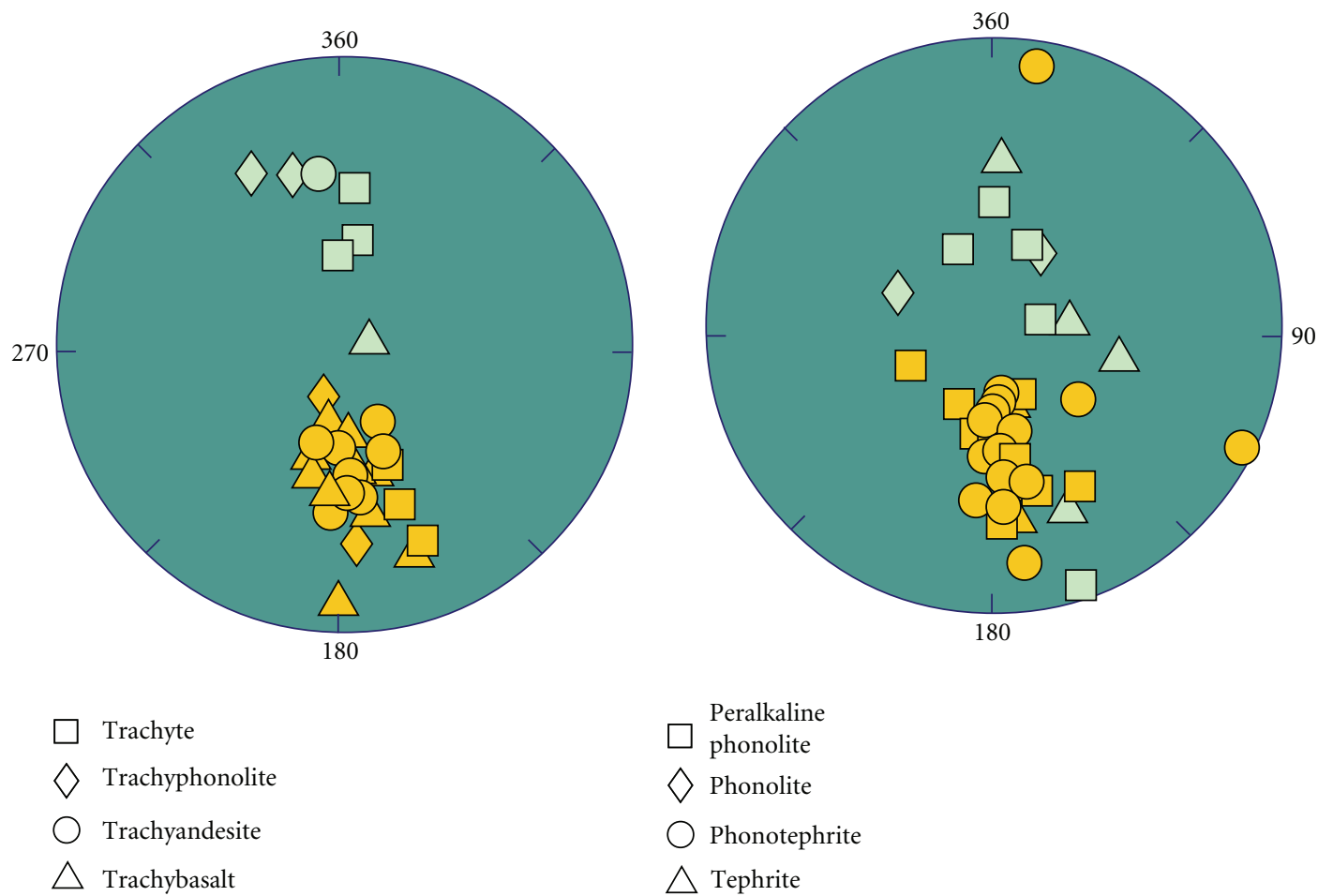
Peralkaline
$\square$ phonolite
$\diamond$ Phonolite
$\bigcirc$ Phonotephrite
$\triangle$ Tephrite

(a)

(b)

Figure 5: Stereographic projection of the site mean paleomagnetic results for the alkaline dykes that occur in the central segment of the Asunción Rift. Note that the majority of the dykes acquired their remanence during a reversed polarity. Data source: [36].

frequent. Interposed between the two main orientations, there are two other less common directions, N-S and E$\mathrm{W}$. On a regional scale, joint and fault systems of similar orientation also occur. The relative chronology between faults and dykes is not always of easy distinction. In some cases, because the interval between the events was relatively short and, in others, because there was an overlap of events, obliterating the former structure. However, in more favorable outcrops, the structural relationships indicate that the dyke intrusion was preceded by synthetic normal and 


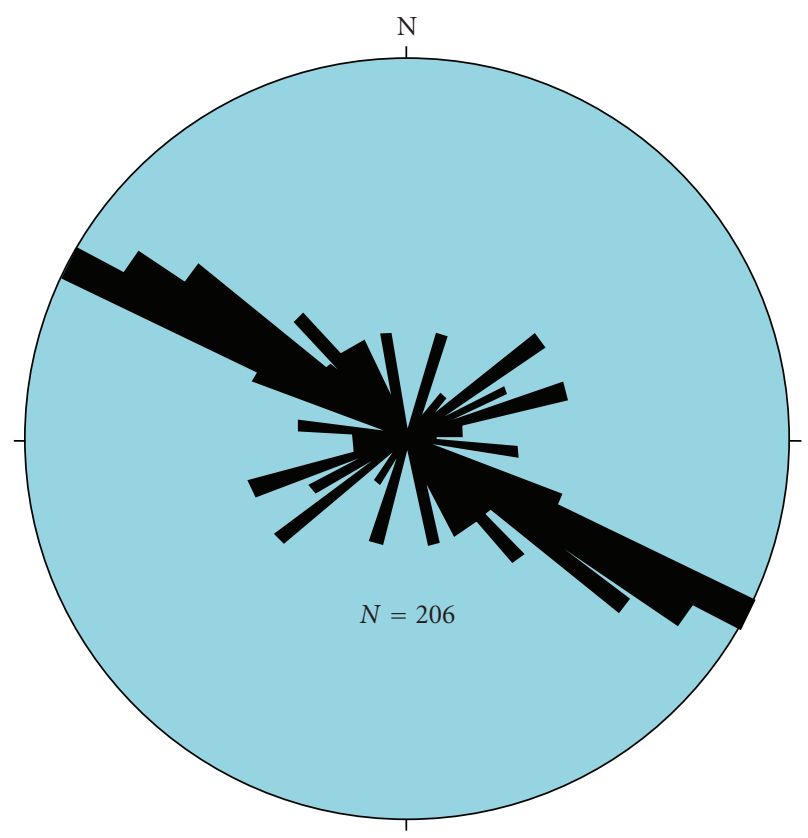

Figure 6: Structural full rose for the alkaline dykes that occur in the central segment of the Asunción Rift.

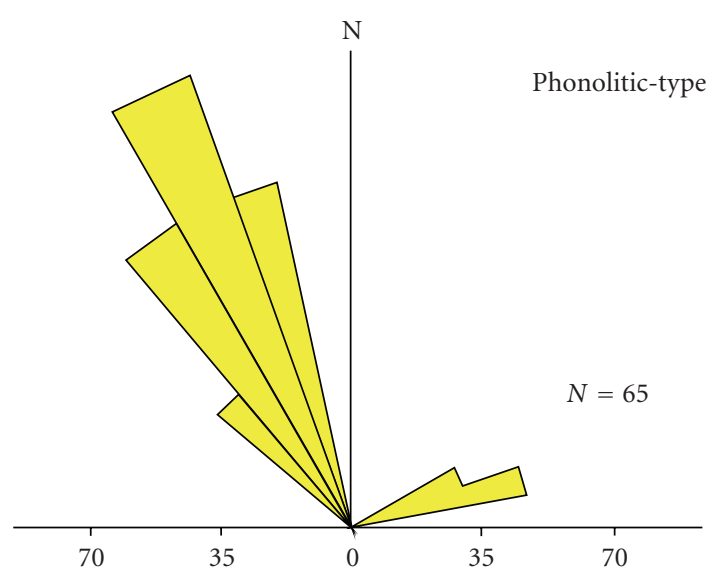

(a)

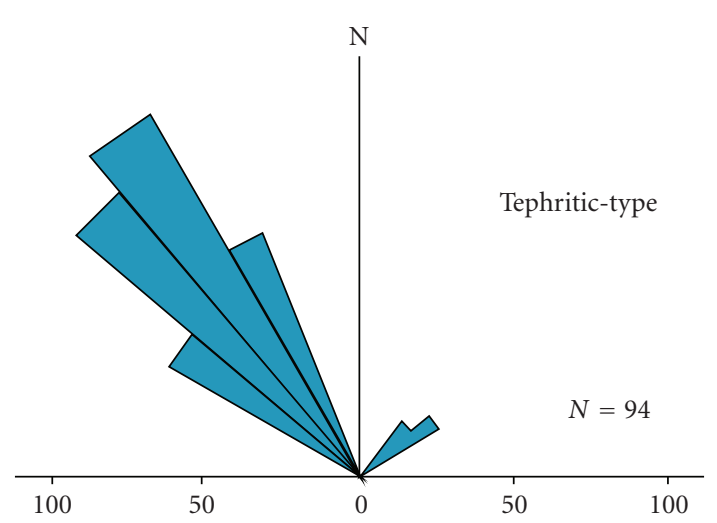

(b)

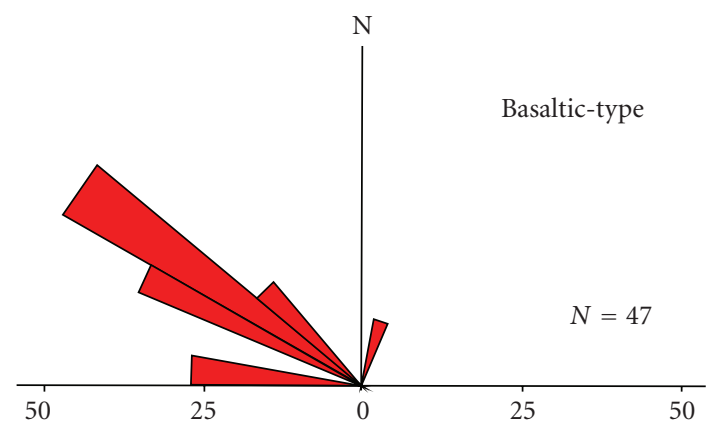

(c)

FIGURE 7: Rose diagrams illustrating the azimuthal orientation for the three main lithological types of alkaline dykes. Note that the mean of the preferential directions indicates a clockwise rotation. 


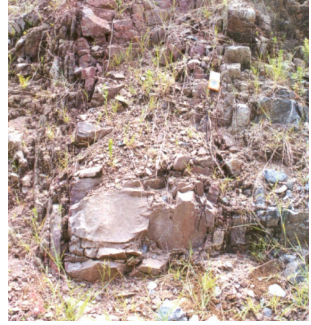

(a)

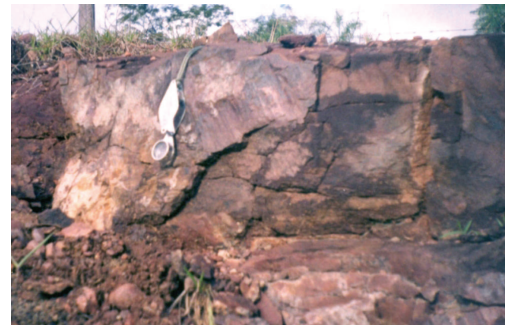

(b)

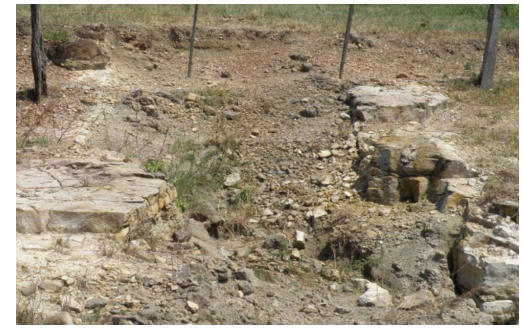

(c)

FIGURE 8: Field relations illustrating the mechanism of emplacement of the dykes: (a) felsic dyke, with a vertical plane orientated according to N25W, evidencing a normal dilatation, (b) intermediate dyke, orientated according to N35W, showing a normal-oblique separation with slickenside surface on the walls of the dyke, and (c) mafic dyke, orientated according to N50W, showing a typical right-lateral opening with oblique movement between the walls.

some strike-slip faulting. Similarly, the determination of the temporal relation of dyke intrusions was based mainly on their cross-cutting relationships. This correlation indicates that basaltic-type predate the tephritic-type and both are cut by phonolitic-type.

Detailed analysis, combining dyke petrography, orientation pattern, and relative chronology, reveals that the basaltic-type is orientated preferentially N50-70W, the tephritic-type N30-60W, and the phonolitic-type N20-45W (Figure 7). Such disposition shows clearly a rotation from WNW toward NNW during the emplacement of the dykes, accompanied by a progressive compositional change of the magma. Field observations are consistent with continuous extensional deformation and right-lateral rotation, which led to a dynamic interrelation between faulting and dyke injection. The absence of internal solid-state deformation in the dykes and the nature of the kinematic indicators present in the walls suggest that the fault generation and the dyke injection would have occurred simultaneously, under an extensional tectonic regime, followed by a short period of progressive shearing, affecting the walls of the partially solidified dykes (Figure 8).

\section{Discussion and Conclusion}

The evolution of the regional stress field is frequently determined by structural analyses of faults, fractures, cleavages, and other microtectonic evidence. Unfortunately, these methods do not permit the precise dating of the specific period in which the stress field has been acting. Igneous rocks, however, can be isotopically dated and frequently display distribution and internal structural patterns related to the crustal stress field at the time of magmatism. Therefore, it is possible to establish an absolute chronology of the variation in the trajectory of the regional paleostressfields [2-5]. This manner, volcanic features are frequently utilized as paleostress indicators, including alignments of volcanoes or ash cones and axes of elongation of volcanic edifices $[37,38]$.

Dykes are also very important kinematic indicators, considering that magma may invade coeval fractures under the action of a regional paleostress-field [39, 40]. Because magma-driven fracturing is preferentially normal to the principal compressive stress $\sigma 1$ (Anderson's prediction), vertical dykes can only be generated when $\sigma 3$ is horizontal, that is, when the regional paleostress of the crust is dominantly extensional $[6,41-45]$.

The Cretaceous alkaline dyke swarms of Central-Eastern Paraguay show a large compositional variation, petrographic facies, and texture, offering important constraints on magma generation and dyke intrusions. Field observations indicate that the dykes represent majorly single vertical intrusions, but composite and multiple intrusions are also recognized.

The wide mineralogical variation and heterogeneous geochemical composition of the dykes require a dynamic generation mechanism during their emplacement, with constant and progressive crystal-liquid fractionation from the magmatic chamber. Such rapid rates of magma generation, in short periods of time, argue that partial melting took place during an extensional tectonic regime and by adiabatic decompression. Many of the dykes contain a significant proportion of primary hydrous minerals (biotite and/or amphibole) suggesting that hydrodynamic force (watervapor pressure) played an important role in the magma generation. As a result of a constant modification of the thermodynamic conditions, the absolute majority of the dykes presents a well-marked change in texture, ranging from holocrystalline to hypocrystalline. In contrast to Komar's prediction [46], no gradual concentration in phenocrysts from the walls to the center or internal structural zoning was observed in those dykes. This fact seems to confirm that flowdifferentiation processes were not an important mechanism responsible for their generation. Thus, it is reasonable to admit that the dykes were emplaced in liquid state and consolidated under very fast cooling rates. In this case, an unstable condition of the flow regime due to variables such as the velocity gradient across the fractures, width of the fractures, magma viscosity, cooling rates, and crystal supply caused different effects on the crystals growth during emplacement and broadly influenced textural variations.

Because thin dyke channels may only last for hours to days (cf. [47]), liquids generated in the mantle and subject to continuous removal toward the surface would 


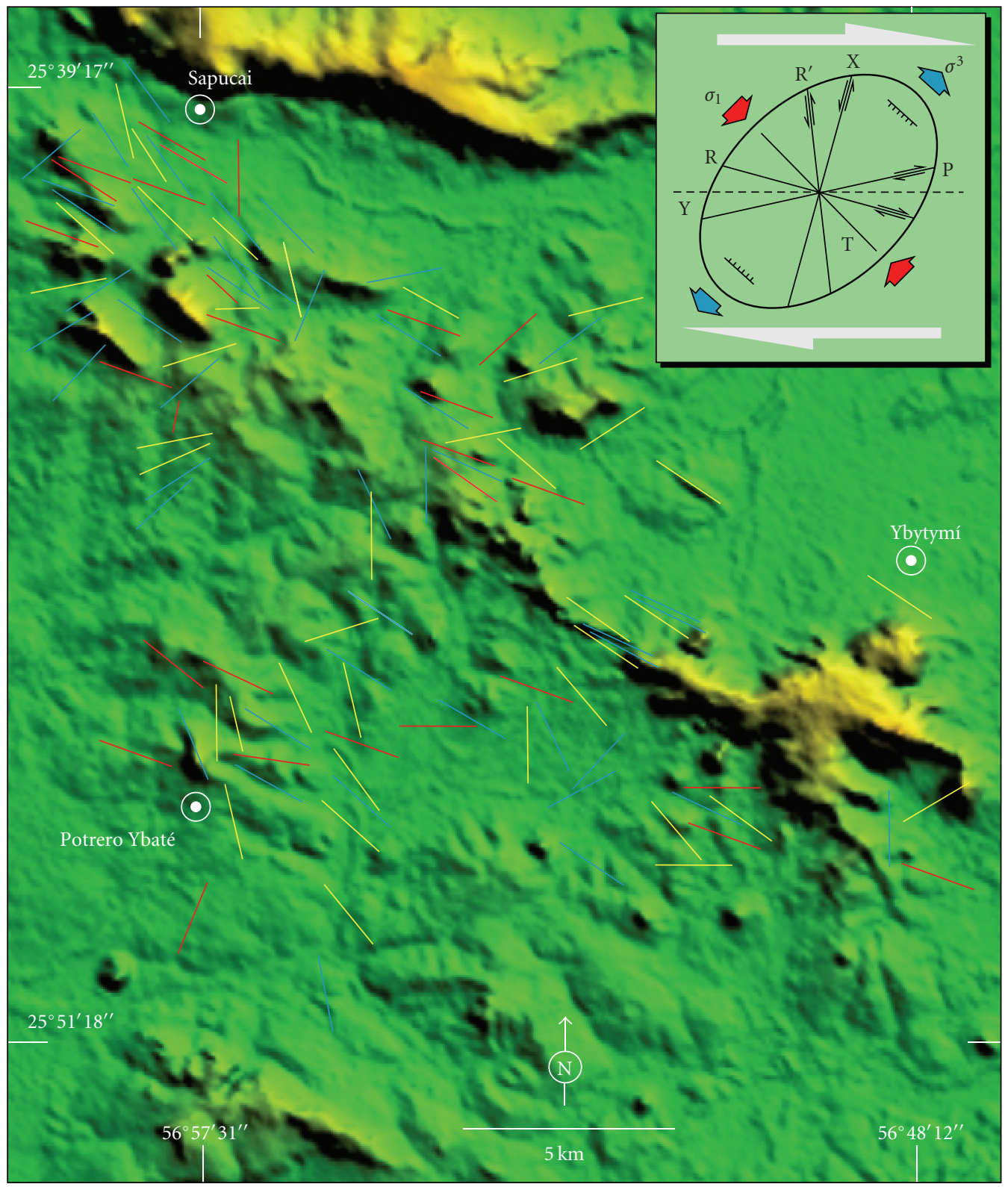

Figure 9: Regional en-échelon distribution and a proposed for the emplacement mechanism of the swarm alkaline dykes (see text for discussion). Schematic Riedel shear model in inset shows theoretical distribution of shear fractures ( R, $\mathrm{R}^{\prime}, \mathrm{P}, \mathrm{X}$ and $\left.\mathrm{Y}\right)$ and tensional fractures (T) in E-W dextral transtensional tectonic regime (cf. [53]). Red and blue arrows indicate shortening axis and extensional axis, respectively. The distinction of dykes is as in Figure 5.

be under the constant influence of the regional stress fields [48-52]. As previously stated, vertical dykes occur as a product of magma-filled fractures when the minimum compressive stress, $\sigma 3$, is perpendicular to the direction of the propagation plane. Such conditions seem to be shown by the field relations of the studied dykes. This assumption is based on the careful examination of the chilled margins of the dykes and their geochemical data, which indicate no obvious significant interaction with the country rocks during magma injection. The walls of the dykes usually display normal dilatation and planar morphology. Only a few bodies show oblique opening, in either a dextral or sinistral sense, with overall extension preferentially orthogonal to the main propagation trajectory of the dykes. Additional evidence, such as the limited length (less than $3 \mathrm{~km}$ ) and narrow width (average of $1-3 \mathrm{~m}$ ) of the sheet-like bodies, the lack of a flux orientation of phenocrysts aligned parallel at the dyke margins, and the en-échelon regional distribution, indicates that vertical injection was more common than lateral magma injection.

The regional N20-70W-trending of the Cretaceous alkaline dykes suggests that the opening fractures and the magma injection were not perturbed significantly by pre-existing lines of weakness in the country rocks. Therefore, the wellexpressed structural fingerprints of the dykes indicate an overall remote stress regime characterized by a NW-trending 


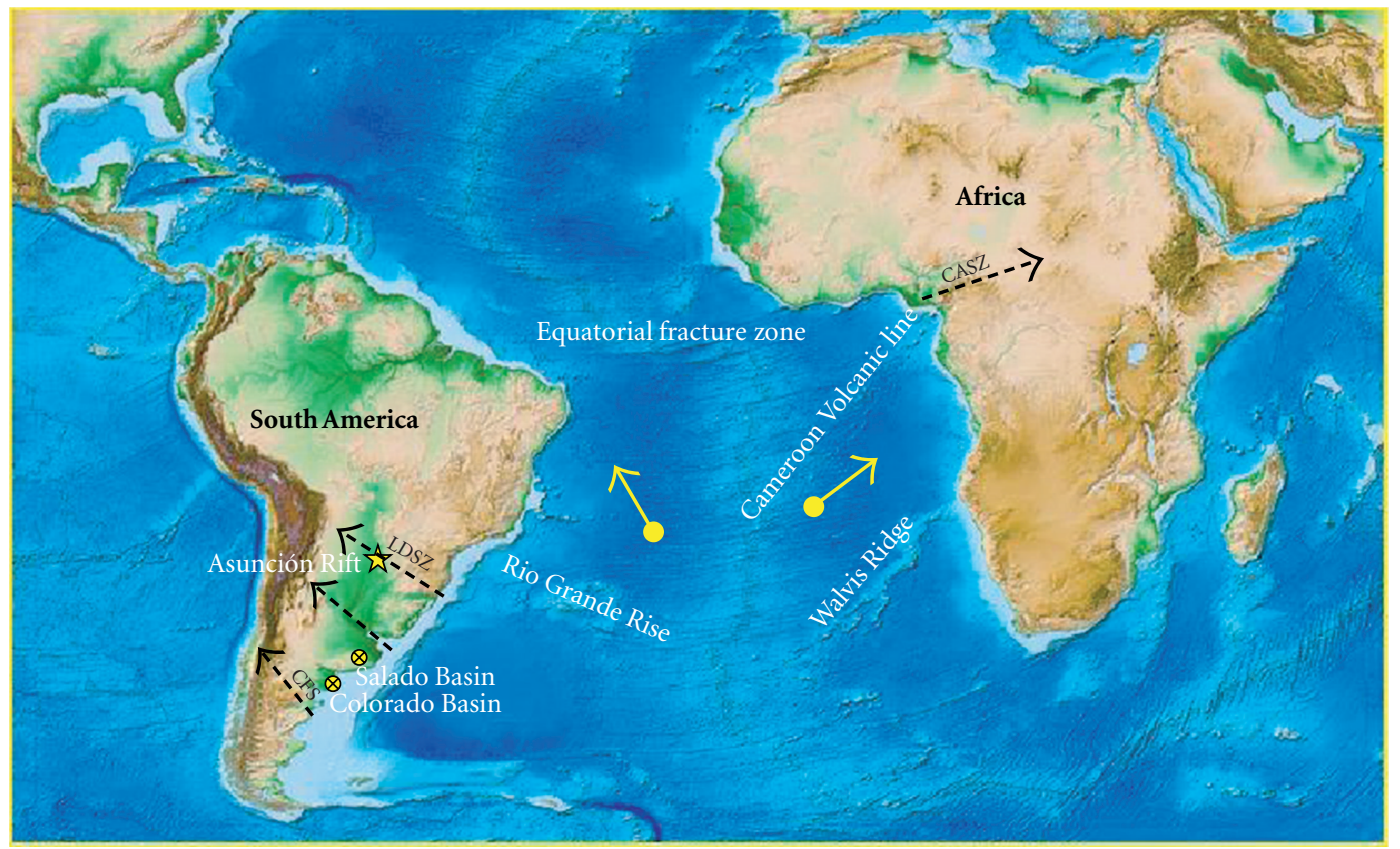

FIGURE 10: Schematic map showing the main larger deformation areas that occurred during the phase of continental breakup. Dashed black arrows: larger dextral shear zone (LDSZ), Gastre fault system (GFS), and central African sinestral shear zone (CASZ). Yellow arrows indicate the plate motion according to Fairhead and Wilson [65]. Note that the Asunción Rift is closely related to the LDSZ. Copyrights of the map for National Geophysical Data Center.

maximum horizontal shortening axis, an NE-oriented maximum horizontal extension axis, and vertical intermediate stress. Slickenside lineation analysis on NNW-sinestral and WNW-dextral conjugate strike-slip faults plane that outline the central segment borders of Asunción Rift (Figure 2) is also compatible with this paleostress orientation [19, 20,22]. Basic criteria, such as the geometry, the orientation, and distribution pattern and the relative chronology of the dykes and regional faults, are widely consistent with an E-W dextral transtensional tectonic regime (Figure 9). In the context of a simple Riedel shear model [53], the basaltic-type dykes were preferentially injected along $\mathrm{R}$ fractures during the initial stage of the deformation. Some tephitic-type dykes also filled in part these fractures. With strain increase, $\mathrm{R}^{\prime}$ followed by $\mathrm{P}$ fractures were generated, which served as conduits for the tephitic-type dykes. Isolated occurrences of phonolitic-type dykes display consistent orientation with this phase of deformation. At an advanced stage of strain, finally, $\mathrm{T}$ fractures, perpendicular to the maximum extension axis, and Y fractures, parallel to the principal displacement zone direction, controlled the intrusion of the phonolitictype dykes. Pattern orientation corresponding to $\mathrm{X}$ fractures is relatively uncommon in the study area. Assuming that the dykes were injected during an event of progressive deformation, the regional distribution in right- and leftstepping en-échelon corresponds to shear fractures ( $R$, $\mathrm{R}^{\prime}$ and $\mathrm{P}$ ) and tensional fractures $(\mathrm{T})$. This framework structural has significant implications with regard to the right-lateral global rotation during dyke intrusions. The local parallel orientation of composite and multiple dykes confirm that the trajectory of the regional paleostress fields was relatively constant at the time of the dyke intrusion.
The relationship between alkaline magmatism associated with the Asunción Rift and basaltic melt generation in the Paraná-Etendeka large igneous province is well recognized [7-9]. Several geological evidence suggest that this episode of instability was occasioned by the lithospheric extension, continental breakup, and mantle plume, provoking a wide range of structural discontinuities, the installation of new sedimentary basins, and the intrusion of numerous alkaline bodies $[8,54-56]$. High-precision ${ }^{40} \mathrm{Ar} /{ }^{39} \mathrm{Ar}$ age determinations indicate that the alkaline magmatism in Eastern Paraguay can be grouped in two main events. The early stage $(\sim 145$ and $138.9 \mathrm{Ma}$, cf. $[7,10])$, located in the north region of the country, was before the first eruption of the flood basalts at $133 \mathrm{Ma}$ (cf. [14, 15]) and a late phase ( $\sim 127$ and 126.4 Ma), concentrated in the Asunción Rift, which coincides with the youngest flood basalts that occur near the Brazilian and Uruguayan shoreline $[7,10]$. In terms of petrogenetic evolution, the Paraná-Etendeka large igneous province and associated alkaline intrusive rocks are commonly interpreted as derived from a similar mantle source region by upwelling Tristan plume [57-59]. Gibson et al. [7] postulated that the late event ( $\sim 127 \mathrm{Ma})$ of Paraguayan alkaline magmatism would be associated with the final phase of the opening of the South Atlantic Ocean. According to Riccomini et al. [60] and Comin-Chiaramonti et al. [10], the regional spatial distribution of the Cretaceous and Tertiary alkaline magmatism along the coast lines of the South Atlantic continental margins and West Africa reveal that major dextral and sinestral shear zones exerted significant control on the alkaline bodies alignment. This evidence suggests an analogous intraplate deformation in both South American and African plates, as previously proposed by Vink 
[61] and Fairhead [39]. In this context, a lateral perturbation of the regional stress intensity induced by the differential motion of the plates at the time of continental breakup would favor the propagation of brittle structures toward the interior of the continents (Figure 10). Finally, the presence of a dextral strike-slip motion along the South American second-order plate boundary, proposed by Unternehr et al. [62] and N. Eyles and C. H. Eyles [63], the south-eastwards migration of Paraná-Etendeka large igneous province and associated alkaline rocks, suggested by Turner et al. [14] and Gibson et al. [7], and the Cabo Frio magmatic lineament [60] are strongly indicative of a global motion towards WNW of the South American plate, consistent with the pattern of orientation documented for the Cretaceous alkaline dykes of the Asunción Rift.

\section{Acknowledgments}

The authors thank Fapesp for financial support (Proc.: 97/01210-4 and Proc. 07/57461-9). They also thank the two anonymous reviewers for their constructive and perceptive commentaries of the manuscript.

\section{References}

[1] H. C. Halls and W. F. Fahrig, "Dyke swarms and continental rifting: some concluding remarks," Geological Association of Canada, vol. 34, pp. 483-492, 1987.

[2] A. J. Parker, P. C. Rickwood, and D. H. Tucker, Mafic Dykes and Emplacement Mechanisms, Balkema, Rotterdam, The Netherlands, 1990.

[3] A. Rubin and D. Pollard, "Dike-induced faulting in rift zones in Iceland and Afar," Geology, vol. 16, pp. 413-417, 1998.

[4] D. D. Pollard, "Elementary fracture mechanics applied to the structural interpretation of dykes," in Mafic Dykes Swarms, H. H. Halls and W. F. Fahrig, Eds., vol. 4, pp. 5-24, Geological Association of Canada, 1987.

[5] P. T. Delaney, D. D. Pollard, J. I. Ziony, and H. Mckee, "Field relation between dykes and joints: emplacement processes and paleostress analysis," Journal of Geophysical Research, vol. 91, pp. 4920-4938, 1986.

[6] E. M. Anderson, The Dynamics of Faulting and Dyke Formation with Applications to Britain, Oliver and Boyd, Edinburg, UK, 1951.

[7] S. A. Gibson, R. N. Thompson, and J. A. Day, "Timescales and mechanisms of plume-lithosphere interactions: ${ }^{40} \mathrm{Ar}-{ }^{39} \mathrm{Ar}$ geochronology and geochemistry of alkaline igneous rocks from the Paraná-Etendeka large igneous province," Earth and Planetary Science Letters, vol. 251, no. 1-2, pp. 1-17, 2006.

[8] P. Comin-Chiaramonti and C. B. Gomes, Eds., Alkaline Magmatism in Central-Eastern Paraguay. Relationships with Coeval Magmatism in Brazil, Edusp-Fapesp, São Paulo, Brazil, 1996.

[9] P. Comin-Chiaramonti and C. B. Gomes, Eds., Mesozoic to Cenozoic Alkaline Magmatism in the Brazilian Platform, Edusp-Fapesp, São Paulo, Brazil, 2005.

[10] P. Comin-Chiaramonti, A. Marzoli, C. B. Gomes et al., "The origin of post-Paleozoic magmatism in Eastern Paraguay," Special Paper of the Geological Society of America, no. 430, pp. 603-633, 2007.
[11] P. R. Renne, M. Ernesto, I. G. Pacca et al., "The age of Paraná flood volcanism, rifting of gondwanaland, and the JurassicCretaceous boundary," Science, vol. 258, no. 5084, pp. 975979, 1992.

[12] P. R. Renne, D. F. Mertz, W. Teixeira, H. Ens, and M. Richards, "Geochronologic constraints on magmatic and tectonic evolution of the Paraná Province," American Geophysical Union Abstract, vol. 74, p. 553, 1993.

[13] P. R. Renne, K. Deckart, M. Ernesto, G. Féraud, and E. M. Piccirillo, "Age of the Ponta Grossa dike swarm (Brazil), and implications to Paraná flood volcanism," Earth and Planetary Science Letters, vol. 144, no. 1-2, pp. 199-211, 1996.

[14] S. Turner, M. Regelous, S. Kelley, C. Hawkesworth, and M. Mantovani, "Magmatism and continental break-up in the South Atlantic: high precision ${ }^{40} \mathrm{Ar}-{ }^{39} \mathrm{Ar}$ geochronology," Earth and Planetary Science Letters, vol. 121, no. 3-4, pp. 333348, 1994.

[15] K. Stewart, S. Turner, S. Kelley, C. Hawkesworth, L. Kirstein, and M. Mantovani, "3-D, ${ }^{40} \mathrm{Ar}-{ }^{39} \mathrm{Ar}$ geochronology in the Paraná continental flood basalt province," Earth and Planetary Science Letters, vol. 143, no. 1-4, pp. 95-109, 1996.

[16] C. B. Gomes, P. Comin-Chiaramonti, V. F. Velázquez, and D. Orué, "Alkaline magmatism in Paraguay: a review," in Alkaline Magmatism in Central-Eastern Paraguay. Relationships with Coeval Magmatism in Brazil, P. Comin-Chiaramonti and C. B. Gomes, Eds., pp. 31-56, Edusp-Fapesp, São Paulo, Brazil, 1996.

[17] V. F. Velázquez, C. B. Gomes, D. Orué, and P. CominChiaramonti, "Magmatismo alcalino do Paraguai: uma revisão e atualização das províncias," Boletim do Instituto de Geociências, vol. 27, pp. 61-79, 1996.

[18] P. Comin-Chiaramonti, A. Cundari, E. M. Piccirillo et al., "Potassic and sodic igneous rocks from Eastern Paraguay: their origin from the lithospheric mantle and genetic relationships with the associated Paraná flood tholeiites," Journal of Petrology, vol. 38, no. 4, pp. 495-528, 1997.

[19] V. F. Velázquez, C. Riccomini, C. B. Gomes, L. Figueredo, and C. Figueredo, "Relações tectônicas do magmatismo alcalino do Paraguai Oriental," Revista do Instituto Geológico, vol. 19, pp. 41-48, 1998.

[20] C. Riccomini, V. F. Velázquez, and C. B. Gomes, "Cenozoic lithospheric faulting in the Asunción Rift, eastern Paraguay," Journal of South American Earth Sciences, vol. 14, no. 6, pp. 625-630, 2001.

[21] J. M. DeGraff, "Late Mesozoic crustal extension and rifting on the western of the Paraná Basin, Paraguay," Geological Society of America, vol. 17, p. 560, 1985.

[22] C. Riccomini, V. F. Velázquez, C. B. Gomes, A. Milan, and A. E. M. Sallun, "The tectonic evolution of the Asunción Rift, Eastern Paraguay," Anais da Academia Brasileira de Ciências, vol. 74, p. 555, 2002.

[23] P. Bitschene, Mesozoischer und Kanozoischer anorogener magmatismus in Ostparaguay: arbeiten zur geologie und petrologie zweier Alkaliprovinzen, Ph.D. Dissertation, Heidelberg University, Heidelberg, Germany, 1987.

[24] J. Berrocal and C. Fernandes, "Seismicity in Paraguay and neighbouring regions," in Alkaline Magmatism in CentralEastern Paraguay, Relationships with Coeval Magmatism in Brazil, P. Comin-Chiaramonti and C. B. Gomes, Eds., pp. 5766, Edusp-Fapesp, São Paulo, Brazil, 1996.

[25] K. L. Hegarty, I. R. Duddy, and P. F. Green, "The thermal history in around the Paraná basin using apatite fission track analysis-implications for hydrocarbon occurrences and basin formation," in Alkaline Magmatism in Central-Eastern 
Paraguay, Relationships with Coeval Magmatism in Brazil, P. Comin-Chiaramonti and C. B. Gomes, Eds., pp. 67-84, Edusp-Fapesp, São Paulo, Brazil, 1996.

[26] P. Comin-Chiaramonti, A. Cundari, C. B. Gomes et al., "Potassic dyke swarm in the Sapucai Graben, eastern Paraguay: petrographical, mineralogical and geochemical outlines," Lithos, vol. 28, no. 3-6, pp. 283-301, 1992.

[27] P. Comin-Chiramonti, A. Cundari, C. B. Gomes et al., "Mineral chemistry and its genetic significance of major and accessory mineral from a potassic dyke swarm in the Sapucai Graben, central-eastern Paraguay," Geochimica Brasiliensis, vol. 4, pp. 175-206, 1990.

[28] P. Comin-Chiramonti, A. Cundari, A. De Min, C. B. Gomes, and V. F. Velázquez, "Magmatism in Eastern Paraguay: occurrence and petrography," in Alkaline Magmatism in CentralEastern Paraguay. Relationships with Coeval Magmatism in Brazil, P. Comin-Chiaramonti and C. B. Gomes, Eds., pp. 103122, Edusp-Fapesp, São Paulo, Brazil, 1996.

[29] M. D. Druecker and S. P. Gay Jr., "Mafic dyke swarms associated with Mesozoic rifting in eastern Paraguay, South America," Geological Association of Canada, vol. 34, pp. 187193, 1987.

[30] A. Cundari and P. Comin-Chiaramonti, "Mineral chemistry of alkaline rocks from the Asunción-Sapucai graben (CentralEastern Paraguay)," in Alkaline Magmatism in Central-Eastern Paraguay. Relationships with Coeval Magmatism in Brazil, P. Comin-Chiaramonti and C. B. Gomes, Eds., pp. 181-193, Edusp-Fapesp, São Paulo, Brazil, 1996.

[31] C. B. Gomes, P. Comin-Chiaramonti, A. De Min et al., "Atividade filoniana associada ao complexo alcalino de Sapukai, Paraguai Oriental," Geochimica Brasiliensis, vol. 3, pp. 93-114, 1989.

[32] A. Castorina, R. Petrini, P. Comin-Chiaramonti, G. Capaldi, and G. Pardini, "Potassic magmatism from the AsuncionSapucai graben, Eastern Paraguay: inferences on mantle sources by Sr-Nd isotopic systematics," in Alkaline Magmatism in Central-Eastern Paraguay. Relationships with Coeval Magmatism in Brazil, P. Comin-Chiaramonti and C. B. Gomes, Eds., pp. 195-206, Edusp-Fapesp, São Paulo, Brazil, 1996.

[33] V. F. Velázquez, C. B. Gomes, and G. Capaldi, "Magmatismo alcalino Mesozóico na porção centro oriental do Paraguai: aspectos geocronológicos," Geochimica Brasiliensis, vol. 6, pp. 23-35, 1992.

[34] J. H. Palmieri, El complejo alcalino de Sapukai (Paraguay Oriental), Ph.D. Dissertation, University of Salamanca, Salamanca, Spain, 1973.

[35] C. B. Gomes, A. Milan, and V. F. Velázquez, "Magmatismo alcalino na porção centro-oriental do Paraguai: novos dados geocronológicos para as rochas das Províncias Central e Assunção," in Proceedings of the 7th Congresso de Geoquímica dos países de lingua portuguesa, vol. 1, pp. 179-183, Revista da Faculdade de Ciências, Universidade Eduardo Mondlane, Maputo, Moçambique, 2003.

[36] M. Ernesto, P. Comin-Chiaramonti, C. B. Gomes, and J. C. Velázquez, "Paleomagnetic data from the central alkaline province, Eastern Paraguay," in Alkaline Magmatism in Central-Eastern Paraguay. Relationships with Coeval Magmatism in Brazil, P. Comin-Chiaramonti and C. B. Gomes, Eds., vol. 123, pp. 238-253, Edusp-Fapesp, São Paulo, Brazil, 1996.

[37] E. D. Jackson and H. R. Shaw, "Stress pattern in central portion of the pacific plate delineated in time by linear volcanic chains," Journal of Geophysical Research, vol. 80, pp. 18161874, 1975.
[38] G. Féraud, C. Giannérini, and R. Camprendon, "Dyke swarms as paleostress indicators in areas adjacent to continental collision zones: examples from the European and northwest Arabian plates," Geological Association of Canada, vol. 34, pp. 273-278, 1987.

[39] J. D. Fairhead, "Mesozoic plate tectonic reconstructions of the central South Atlantic Ocean: the role of the West and Central African rift system," Tectonophysics, vol. 155, no. 1-4, pp. 181191, 1988.

[40] A. F. Glazner, J. M. Bartley, and B. S. Carl, "Oblique opening and noncoaxial emplacement of the Jurassic Independence dike swarm, California," Journal of Structural Geology, vol. 21, no. 10 , pp. 1275-1283, 1999.

[41] J. R. Lister, "Fluid-mechanical models of crack propagation and their application to magma transport in dykes," Journal of Geophysical Research, vol. 96, no. 6, p. 10, 1991.

[42] W. F. Fahrig, K. W. Christie, E. H. Chown, D. Janes, and N. Machado, "The tectonic significance of some basic dyke swarms in the Canadian Superior province with special reference to the geochemistry and paleomagnetism of the Mistassini swarm, Quebec, Canada," Canadian Journal of Earth Sciences, vol. 23, no. 2, pp. 238-253, 1986.

[43] W. F. Fahrig, "The tectonic setting of continental mafic dyke swarms: failed arm and early passive margin," Geological Association of Canada, vol. 34, pp. 331-348, 1987.

[44] R. E. Ernst, E. B. Grosfils, and D. Mège, "Giant dike swarms: earth, Venus, and Mars," Annual Review of Earth and Planetary Sciences, vol. 29, pp. 489-534, 2001.

[45] G. Hou, T. M. Kusky, C. Wang, and Y. Wang, "Mechanics of the giant radiating Mackenzie dyke swarm: a paleostress field modeling," Journal of Geophysical Research, vol. 115, no. 2, Article ID B02402, pp. 1-14, 2010.

[46] P. D. Komar, "Phenocryst interactions and the velocity profile of magma flowing through dikes or sills," Geological Society American Bulletin, vol. 83, pp. 1336-1342, 1976.

[47] C. Cadman and J. Tarney, "Intrusion and crystallization features in Proterozoic dyke swarms," in Mafic Dykes and Emplacement Mechanisms, A. J. Parker, P. C. Rickwood, and D. H. Tucker, Eds., pp. 13-24, Balkema, Rotterdam, The Netherlands, 1990.

[48] A. Gudmundsson, "Dyke emplacement at divergent plate boundaries," in Mafic Dykes and Emplacement Mechanisms, A. J. Parker, P. C. Rickwood, and D. H. Tucker, Eds., pp. 47-62, Balkema, Rotterdam, The Netherlands, 1990.

[49] H. C. Halls, "The importance and potential of mafic dyke swarms in studies of geodynamic processes," Geoscience Canada, vol. 9, no. 3, pp. 145-154, 1982.

[50] W. R. A. Baragar, R. E. Ernst, L. Hulbert, and T. Peterson, "Longitudinal petrochemical variation in the Mackenzie dyke swarm, Northwestern Canadian shield," Journal of Petrology, vol. 37, no. 2, pp. 317-359, 1996.

[51] E. B. Grosfils and J. W. Head, "The global distribution of giant radiating dike swarms on Venus: implications for the global stress state," Geophysical Research Letters, vol. 21, no. 8, pp. 701-704, 1994.

[52] A. Gudmundsson, "Surface stresses associated with arrested dykes in rift zones," Bulletin of Volcanology, vol. 65, no. 8, pp. 606-619, 2003.

[53] G. Dresen, "Stress distribution and the orientation of Riedel shears," Tectonophysics, vol. 188, no. 3-4, pp. 239-247, 1991.

[54] F. F. M. D. Almeida, B. B. D. Brito Neves, and C. D. R. Carneiro, "The origin and evolution of the South American platform," Earth Science Reviews, vol. 50, no. 1-2, pp. 77-111, 2000. 
[55] D. Peate, "The Parana-Etendeka Province," in Large Igneuos Province: Continetal, Oceanic, Planetary Flood Volcanism, J. Mahoney and M. F. Coffin, Eds., pp. 217-245, American Geophyscial Union, Washington, DC, USA, 1992.

[56] C. J. Hawkesworth, "Paraná magmatism and the opening of the South Atlantic," Geological Society of London Special Publication, vol. 68, pp. 221-240, 1992.

[57] A. Ewart, S. C. Milner, R. A. Armstrong, and A. R. Duncan, "Etendeka volcanism of the Goboboseb Mountains and Messum Igneous Complex, Namibia. Part I: geochemical evidence of early cretaceous Tristan plume melts and the role of crustal contamination in the Paraná-Etendeka CFB," Journal of Petrology, vol. 39, no. 2, pp. 191-225, 1998.

[58] S. A. Gibson, R. N. Thompson, O. H. Leonardos, A. P. Dickin, and J. G. Mitchell, "The limited extent of plumelithosphere interactions during continental flood-basalt genesis: geochemical evidence from Cretaceous magmatism in southern Brazil," Contributions to Mineralogy and Petrology, vol. 137, no. 1-2, pp. 147-169, 1999.

[59] R. N. Thompson, S. A. Gibson, A. P. Dickin, and P. M. Smith, "Early cretaceous basalt and picrite dykes of the Southern Etendeka Region, NW Namibia: windows into the role of the Tristan mantle plume in Paraná-Etendeka magmatism," Journal of Petrology, vol. 42, no. 11, pp. 2049-2081, 2001.

[60] C. Riccomini, V. F. Velázquez, and C. B. Gomes, "Tectonic controls of the Mesozoic and Cenozoic alkaline magmatism in central-southeastern Brazilian Platform," in Mesozoic to Cenozoic Alkaline Magmatism in the Brazilian Platform, P. Comin-Chiaramonti and C. B. Gomes, Eds., vol. 123, pp. 3156, Edusp-Fapesp, São Paulo, Brazil, 2005.

[61] G. E. Vink, "Continental rifting and the implications for plate tectonic reconstructions," Journal of Geophysical Research, vol. 87, no. 13, pp. 10677-10688, 1982.

[62] P. Unternehr, D. Curie, J. L. Olivet, J. Goslin, and P. Beuzart, "South Atlantic fits and intraplate boundaries in Africa and South America," Tectonophysics, vol. 155, no. 1-4, pp. 169-179, 1988.

[63] N. Eyles and C. H. Eyles, "Glacial geologic confirmation of an intraplate boundary in the Parana basin of Brazil," Geology, vol. 21, no. 5, pp. 459-462, 1993.

[64] H. De La Roche, J. Leterrier, P. Grancalude, and M. Marchal, "A classification of volcanic and plunotic rocks using R1-R2 diagram and major elements analyses. Its relationship with currents nomenclature," Chemical Geology, vol. 29, pp. 183210, 1980.

[65] J. D. Fairhead and M. Wilson, "Sea-floor spreading and deformation processes in the South Atlantic Ocean: are hot spots needed?" 2004, http://www.mantleplumes.org/SAtlantic.html. 

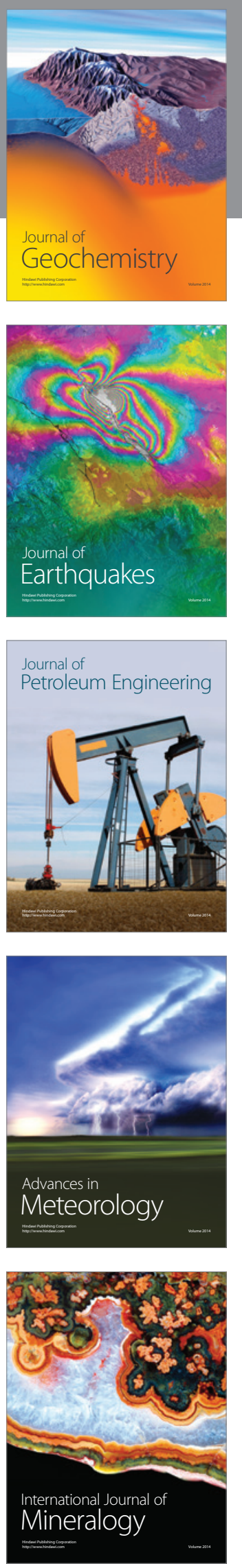
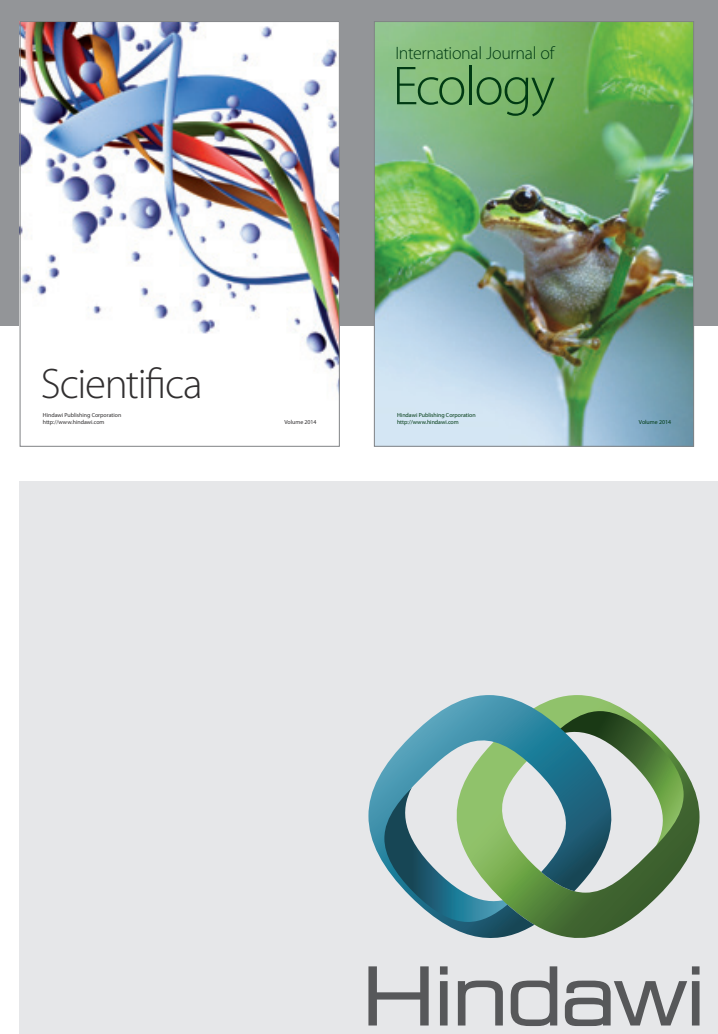

Submit your manuscripts at http://www.hindawi.com
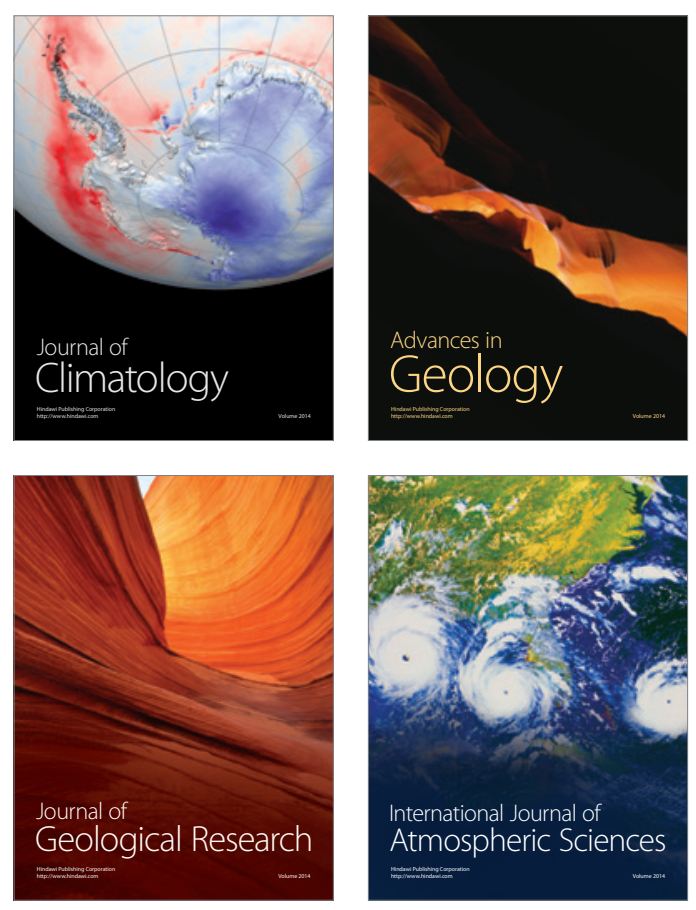
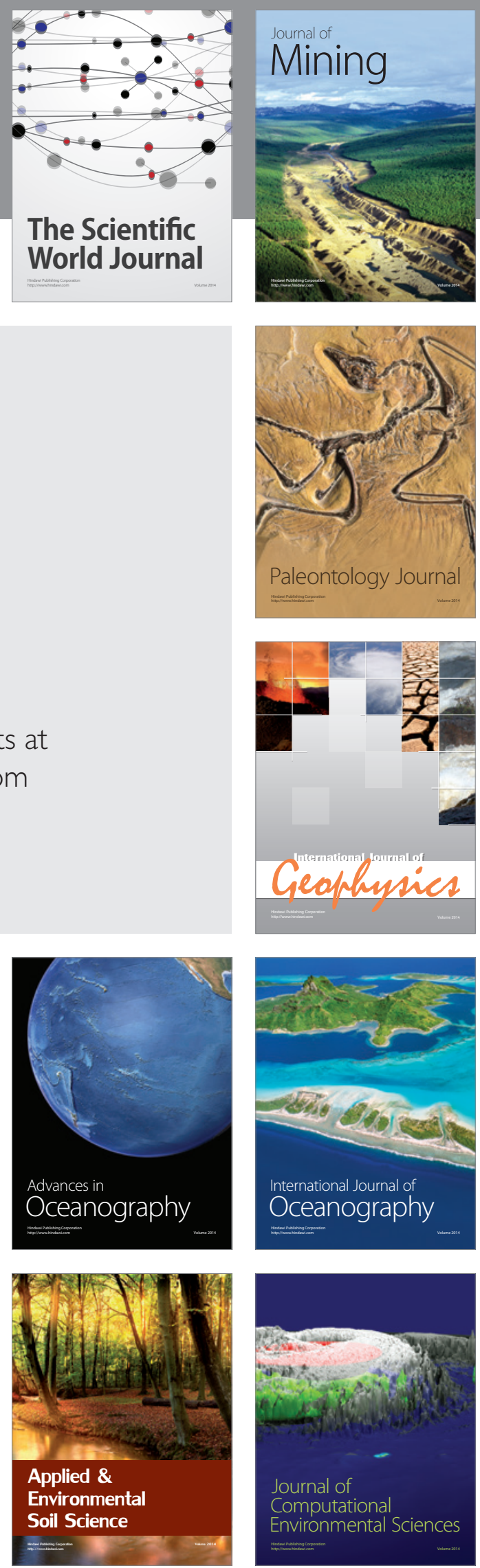